\title{
A POSTERIORI ERROR ESTIMATION FOR $h p$-ADAPTIVITY FOR FOURTH-ORDER EQUATIONS
}

\author{
PETER K. MOORE AND MARINA RANGELOVA
}

\begin{abstract}
A posteriori error estimates developed to drive $h p$-adaptivity for second-order reaction-diffusion equations are extended to fourth-order equations. A $C^{1}$ hierarchical finite element basis is constructed from HermiteLobatto polynomials. A priori estimates of the error in several norms for both the interpolant and finite element solution are derived. In the latter case this requires a generalization of the well-known Aubin-Nitsche technique to time-dependent fourth-order equations. We show that the finite element solution and corresponding Hermite-Lobatto interpolant are asymptotically equivalent. A posteriori error estimators based on this equivalence for solutions at two orders are presented. Both are shown to be asymptotically exact on grids of uniform order. These estimators can be used to control various adaptive strategies. Computational results for linear steady-state and timedependent equations corroborate the theory and demonstrate the effectiveness of the estimators in adaptive settings.
\end{abstract}

\section{INTRODUCTION}

A significant effort has been made in developing adaptive finite element methods for the solution of second-order parabolic and elliptic partial differential equations 2. 5. Less attention has been devoted to fourth-order problems. Nevertheless, many important physical systems are modeled by fourth-order equations. These include the biharmonic equation, the Cahn-Hilliard equation [9, 16] and multicomponent Cahn-Hillard system [14, the Extended Fisher-Kolmogorov equation 6. 12, the Kuramoto-Sivashinsky equation [11, 18, 30, 32, and the Generalized Swift-Hohenberg equation [8, 17]. In these examples the solutions are smooth, suggesting that high-order approaches are advantageous.

One approach to solving fourth-order equations is to reduce them to a secondorder system and utilize well-established adaptive methods for second-order equations. Adaptive finite element methods for second-order systems come in several varieties. Two of the most common are grid-refinement ( $h$-refinement) and order variation ( $p$-refinement) [5]. A combination of these, i.e., $h p$-refinement has been shown to be very effective in several settings 13,20 .

Potential drawbacks of this strategy include measuring errors in less natural norms and a doubling of the number of unknowns. One approach to reducing the number of unknowns is by using discontinuous Galerkin [10] or interior penalty

Received by the editor December 11, 2007 and, in revised form, April 14, 2009.

2000 Mathematics Subject Classification. Primary 65M60; Secondary 65M15.

Key words and phrases. A posteriori error estimation, fourth-order equations, adaptivity.

(C)2009 American Mathematical Society Reverts to public domain 28 years from publication 
methods 33. Alternatively the fourth-order problems can be solved directly enabling us to compute solutions in $H^{2}$. To that end we consider the basis proposed by Adjerid [1] constructed from Hermite-Lobatto polynomials. This basis has several desirable properties. It involves less than half the number of unknowns of the second-order system. The finite element solution is in $C^{1}$. The basis is hierarchical in that increasing the order on an element involves one additional basis function. Most importantly the Hermite-Lobatto interpolant possesses the asymptotic equivalence property, that is, the finite element and interpolant errors converge at the same rate with the same constant in the $H^{2}$ norm. Asymptotic equivalence guarantees that error estimates that converge to the true error as the grid is refined can be constructed [3, 23, 24, 38, 39.

These estimates are global in the sense that they approximate the error over the domain. They are used to decide when to adapt the grid. How to adapt the grid, on the other hand, depends on estimates of the local error. Error indicators on each element are typically computed as the elemental contributions to the global error estimator. In the approach taken by Adjerid for even-order elements in two space dimensions [1] and followed here, error indicators are obtained by solving a local problem on each element. These suffice for determining $h$ - and $p$-refinement. The order selection algorithm for $h p$-adaptivity adopted by us also requires estimates of the error one order higher than the current order. This is accomplished by solving a second local problem on each element. These two indicators are natural extensions of effective techniques developed for second-order equations 25 .

The error estimator and element error indicators proposed, in conjunction with $h-, p$ - or $h p$-adaptivity can be utilized to solve both steady-state and time-dependent problems. To that end consider fourth-order steady-state equations of the form

$$
\begin{aligned}
\frac{d^{2}}{d x^{2}}\left(\rho(x) \frac{d^{2} u}{d x^{2}}\right)-\frac{d}{d x}\left(\mu(x) \frac{d u}{d x}\right)+\kappa(x) u & =f(x), x \in \Omega \equiv(a, b), \\
u(a)=u(b)=0, & \frac{d u}{d x}(a)=\frac{d u}{d x}(b)=0,
\end{aligned}
$$

and comparable time-dependent equations

$$
\begin{gathered}
u_{t}+\left(\rho(x) u_{x x}\right)_{x x}-\left(\mu(x) u_{x}\right)_{x}+\kappa(x) u=f(x, t), x \in \Omega \equiv(a, b), t \in(0, T], \\
u(a, t)=u(b, t)=0, \quad \frac{d u}{d x}(a, t)=\frac{d u}{d x}(b, t)=0, \\
u(x, 0)=u_{0}(x), x \in \Omega,
\end{gathered}
$$

where

$$
\rho(x)>0, \mu(x) \geq 0, \kappa(x) \geq 0 .
$$

Throughout we assume that (1)-(2) and (3)-(5) have unique solutions. In the former case we also assume $u \in H_{0}^{p+5}(\Omega)$ while in the latter $u \in H_{0}^{p+5}(\Omega) \times C_{0}^{\infty}[0, T]$ for some time $T$. These regularity assumptions are needed in our proofs but are not the primary focus of this paper. Our main concern is in developing estimators that will result in reliable and efficient codes for problems with smooth solutions. Equations (11)-(2) and (3)-(5) are discretized in space using the finite element method. For (3)-(15) the resulting system of ordinary differential equations is integrated in time using the differential-algebraic code dassl [7. This corresponds to a method-of-lines (MOL) approach. 
In section 2 the Hermite-Lobatto polynomials are introduced and important properties are derived. A priori estimates for the Hermite-Lobatto interpolant on a canonical element $\Delta=[m-h / 2, m+h / 2]$ and on $\Omega$ are obtained in section 3 . Comparable results for the associated finite element solutions and relations between these a priori error estimates are also proved. This requires an extension of the Aubin-Nitsche technique [15] to fourth-order time-dependent problems. In section 4 a posteriori error estimates for the finite element solutions of both fourth-order problems are presented. Estimates for a finite element solution one order higher are also obtained in this section. In both cases convergence of the estimates to the true error is proved for grids of uniform order. This represents an important first step in obtaining proofs in a more general setting. Finally a brief description of how the theory is applied to guide the three adaptive strategies is presented. Section 5 contains the computational results. Estimator accuracy is confirmed for a model linear steady-state problem. We also provide numerical evidence that the error estimator and indicators can effectively drive the adaptive strategies along with some comparisons between them for steady-state and time-dependent equations. Some conclusions are presented in section 6 .

\section{Preliminaries}

Throughout this paper the Hermite-Lobatto polynomials on $\Delta=[m-h / 2, m+$ $h / 2$ ] are given by

$$
\Phi_{p}(x ; h ; m)=\sqrt{\frac{2 p-3}{2}} \int_{-1}^{2(x-m) / h} \int_{-1}^{r} P_{p-2}(s) d s d r, p \geq 4
$$

and in monic form by

$$
\psi_{p}(x ; h ; m)=\prod_{i=1}^{p}\left(x-\Gamma_{i}\right)=\sum_{i=0}^{\left[\frac{p}{2}\right]} \hat{a}_{p-2 i}^{p} x^{p-2 i}, p \geq 4
$$

where $P_{p-2}(x)$ is the Legendre polynomial of degree $p-2$ and the $\Gamma_{i}, i=1, \ldots, p$, are the roots of $\Phi_{p}(x ; h ; m)$. To form a basis for the space of polynomials of degree $p$ on $\Delta$ the functions $\Phi_{p}(x ; h ; m), p \geq 4$, of (7) are augmented by the cubic polynomials,

$$
\begin{aligned}
\Phi_{0}(x ; h ; m) & =\frac{2}{h^{3}}\left(x-\left(m+\frac{h}{2}\right)\right)^{2}(x-(m-h)), \\
\Phi_{1}(x ; h ; m) & =\frac{1}{h^{2}}\left(x-\left(m+\frac{h}{2}\right)\right)^{2}\left(x-\left(m-\frac{h}{2}\right)\right), \\
\Phi_{2}(x ; h ; m) & =\frac{2}{h^{3}}\left(x-\left(m-\frac{h}{2}\right)\right)^{2}(m+h-x), \\
\Phi_{3}(x ; h ; m) & =\frac{1}{h^{2}}\left(x-\left(m-\frac{h}{2}\right)\right)^{2}\left(x-\left(m+\frac{h}{2}\right)\right) .
\end{aligned}
$$

We will need the following properties of $\psi_{p}(x ; h ; m)$ and its derivatives: 
Lemma 1. On $\Delta$,

$$
\begin{aligned}
& \psi_{p}(x ; h ; m)=\frac{h^{p}(p-3) ! p !}{8(2 p-5) !} \sqrt{\frac{2}{2 p-3}} \Phi_{p}(x ; h ; m) \\
& \psi_{p}(x ; h ; m)=\frac{h^{p} p !(p-1) !}{2(2 p-1) !}\left(P_{p}(2(x-m) / h)-\frac{2(2 p-3)}{2 p-5} P_{p-2}(2(x-m) / h)\right. \\
&\left.+\frac{2 p-1}{2 p-5} P_{p-4}(2(x-m) / h)\right)
\end{aligned}
$$

(15) $\psi_{p}^{\prime}(x ; h ; m)=\frac{h^{p-1} p !(p-2) !}{2(2 p-3) !}\left(P_{p-1}(2(x-m) / h)-P_{p-3}(2(x-m) / h)\right)$,

(16) $\psi_{p}^{\prime \prime}(x ; h ; m)=\frac{h^{p-2} p !(p-1) !}{(2 p-4) !} P_{p-2}(2(x-m) / h)$,

$$
\begin{aligned}
& \text { (17) } \int_{m-h / 2}^{m+h / 2} \psi_{p}^{2}(x ; h ; m) d x=\frac{3 h^{2 p+1}(2 p-3)}{8(2 p+1)(2 p-1)(2 p-5)(2 p-7)}\left(\frac{p !(p-2) !}{(2 p-2) !}\right)^{2}, \\
& \text { (18) } \int_{m-h / 2}^{m+h / 2}\left(\psi_{p}^{\prime}(x ; h ; m)\right)^{2} d x=\frac{h^{2 p-1}(2 p-3)}{2(2 p-1)(2 p-5)}\left(\frac{p !(p-2) !}{(2 p-3) !}\right)^{2}, \\
& \text { (19) } \int_{m-h / 2}^{m+h / 2}\left(\psi_{p}^{\prime \prime}(x ; h ; m)\right)^{2} d x=\frac{h^{2 p-3}}{2 p-3}\left(\frac{p !(p-2) !}{(2 p-4) !}\right)^{2} \\
& \text { (20) } \psi_{p+1}(x)=(x-m) \psi_{p}(x)-\frac{h^{2} p(p-4)}{4(2 p-3)(2 p-5)} \psi_{p-1}(x), \\
& \text { (21) } \max _{x \in \Delta}\left|\psi_{p}^{\prime \prime}(x ; h ; m)\right| \leq C(p) h^{p-2} .
\end{aligned}
$$

Proof. The first equality is obtained from (7) and the properties of Legendre polynomials 28]

$$
P_{p-2}(x)=\frac{2^{p-2}\left(\frac{1}{2}\right)_{p-2} x^{p-2}}{(p-2) !}+R_{p-4}(x),
$$

where $R_{p-4}(x)$ is a polynomial of degree $p-4$ and the factorial function $(1 / 2)_{p-2}$ is defined in [28. From [22] the Lobatto polynomial $B_{p}(x)$ satisfies

$$
B_{p}^{\prime}(x)=-\frac{2 p(p-1)}{h} P_{p-1}(2(x-m) / h) .
$$

Thus

$$
-\frac{B_{p}^{\prime}(x)}{p(p-1)}+\frac{B_{p-2}^{\prime}(x)}{(p-2)(p-3)}=\frac{2}{h}\left(P_{p-1}(2(x-m) / h)-P_{p-3}(2(x-m) / h)\right) .
$$

The properties of Legendre polynomials and (23) imply that

$$
\left(P_{p-1}(2(x-m) / h)-P_{p-3}(2(x-m) / h)\right)=-\frac{(2 p-3)}{(p-1)(p-2)} B_{p-1}(x) .
$$

Combining (24) and (25) and integrating over $[m-h / 2, x]$ yields (26)

$$
\Phi_{p}(x ; h ; m)=-\sqrt{\frac{2 p-3}{2}}\left(\frac{1}{p(p-1)(2 p-3)} B_{p}(x)-\frac{1}{(p-2)(p-3)(2 p-3)} B_{p-2}(x)\right) .
$$


Then (14) follows from (26) together with (13) and

$$
B_{p}(x)=-\frac{p(p-1)}{2 p-1}\left(P_{p}(2(x-m) / h)-P_{p-2}(2(x-m) / h)\right),
$$

from 22]. Differentiating (14) and using the properties of Legendre polynomials gives (15). Equation (16) follows similarly by differentiating (15). Formulas (17)(19) are obtained by integrating (14)-(16) and using the properties of Legendre polynomials.

Integrating (23) twice and using (7) yields

$$
\int_{m-h / 2}^{x} B_{p}(s) d s=-\frac{p(p-1) h}{2} \sqrt{\frac{2}{2 p-1}} \Phi_{p+1}(x ; h ; m) .
$$

From [22] we obtain

$$
B_{p}(x)=\frac{(2 p-3)}{(p-2)} \frac{2(x-m)}{h} B_{p-1}(x)-\frac{p-1}{p-2} B_{p-2}(x) .
$$

Integrating (29) gives

$$
\begin{aligned}
\int_{m-h / 2}^{x} B_{p}(s) d s= & \frac{2(2 p-3)}{h(p-2)}\left((x-m) \int_{m-h / 2}^{x} B_{p-1}(s) d s\right. \\
+ & \left.\frac{h}{2} \sqrt{\frac{2}{2 p-3}}(p-1)(p-2) \int_{m-h / 2}^{x} \Phi_{p}(s ; h ; m) d s\right) \\
& -\frac{p-1}{p-2} \int_{m-h / 2}^{x} B_{p-2}(s) d s
\end{aligned}
$$

where we have integrated the second term by parts and then used (28). Substituting (26) into the second term on the right in (30) and applying (28) followed by (13) yields (20). Equation (21) follows from (16) and the properties of Legendre polynomials.

\section{A PRIORI ERROR ESTIMATES}

Two interpolants of $u$ are constructed in this section, one on the interval $\Delta$ and the other on the domain $\Omega$. Consider first a function $u \in C^{\infty}(\Delta)$ and corresponding Hermite-Lobatto interpolant $U_{I, \Delta}$ of the form

$$
U_{I, \Delta}(x)=\sum_{i=0}^{p} \bar{U}_{i} \Phi_{i}(x ; h ; m) .
$$

The coefficients $\bar{U}_{i}, i=0, \ldots, p$, are determined by requiring that

$$
U_{I, \Delta}\left(\Gamma_{j}\right)=u\left(\Gamma_{j}\right), i=2, \ldots, p, U_{I, \Delta}^{\prime}\left(\Gamma_{i}\right)=u^{\prime}\left(\Gamma_{i}\right), i=1, p+1,
$$

with $\Gamma_{1}=\Gamma_{2}=m-h / 2$ and $\Gamma_{p}=\Gamma_{p+1}=m+h / 2$. Additionally let $\pi^{\Delta}$ be the linear operator that projects functions in $C^{\infty}(\Delta)$ onto the space of polynomials of degree $p$ on $\Delta$ according to (32). The next lemma provides a basis for the Hermite-Lobatto interpolant on $\Delta$.

Lemma 2. Let

(33) $\Gamma_{0}=m-\frac{h}{2}+\left(-\frac{2}{h}+\sum_{i=3}^{p-1} \frac{1}{-\frac{h}{2}-\Gamma_{i}}\right)^{-1}, \Gamma_{p+2}=m+\frac{h}{2}+\left(\frac{2}{h}+\sum_{i=3}^{p-1} \frac{1}{\frac{h}{2}-\Gamma_{i}}\right)^{-1}$, 
and

$$
\begin{gathered}
L_{1}(x)=\frac{\prod_{j=2}^{p+1}\left(x-\Gamma_{j}\right)}{\prod_{j=3}^{p+1}\left(m-\frac{h}{2}-\Gamma_{j}\right)}, \quad L_{2}(x)=\frac{\left(x-\Gamma_{0}\right) \prod_{j=3}^{p+1}\left(x-\Gamma_{j}\right)}{\left(m-\frac{h}{2}-\Gamma_{0}\right) \prod_{j=3}^{p+1}\left(m-\frac{h}{2}-\Gamma_{j}\right)}, \\
L_{i}(x)=\prod_{j=1, j \neq i}^{p+1} \frac{x-\Gamma_{j}}{\Gamma_{i}-\Gamma_{j}}, i=3, \ldots p-1, \\
L_{p+1}(x)=\frac{\prod_{j=1}^{p}\left(x-\Gamma_{j}\right)}{\prod_{j=1}^{p-1}\left(m+\frac{h}{2}-\Gamma_{j}\right)}, \quad L_{p}(x)=\frac{\left(x-\Gamma_{p+2}\right) \prod_{j=1}^{p-1}\left(x-\Gamma_{j}\right)}{\left(m+\frac{h}{2}-\Gamma_{p+2}\right) \prod_{j=1}^{p-1}\left(m+\frac{h}{2}-\Gamma_{j}\right)} .
\end{gathered}
$$

Then

$$
U_{I, \Delta}(x)=\sum_{i=2}^{p} u\left(\Gamma_{i}\right) L_{i}(x)+u^{\prime}(m-h / 2) L_{1}(x)+u^{\prime}(m+h / 2) L_{p+1}(x) .
$$

Remark. $\Gamma_{0} \in[m-h, m-h / 2)$ and $\Gamma_{p+2} \in(m+h / 2, m+h]$ with $\Gamma_{0}=m-h$ and $\Gamma_{1}=m+h$ only when $p=3$.

Proof. The result follows from (31)-(32) since $L_{i}\left(\Gamma_{j}\right)=\delta_{i j}, i, j=2, \ldots, p, L_{i}^{\prime}\left(\Gamma_{1}\right)=$ $L_{i}^{\prime}\left(\Gamma_{p+1}\right)=0, i=2, \ldots, p, L_{1}\left(\Gamma_{j}\right)=L_{p+1}\left(\Gamma_{j}\right)=0, j=2, \ldots, p, L_{1}^{\prime}\left(\Gamma_{1}\right)=$ $L_{p+1}^{\prime}\left(\Gamma_{p+1}\right)=1$ and $L_{1}^{\prime}\left(\Gamma_{p+1}\right)=L_{p+1}^{\prime}\left(\Gamma_{1}\right)=0$.

The derivation of the a priori and a posteriori error estimates depends on the series expansion of the error in the Hermite-Lobatto interpolant given in the following theorem.

Theorem 3. Let $u \in C^{\infty}(\Delta)$ and $U_{I, \Delta}$ be defined by (31)-(32). Then if

$$
\begin{aligned}
& v(x)=\sum_{i=0}^{p+q+1} \frac{(x-m)^{i}}{i !} u^{(i)}(m), \\
& u(x)-U_{I, \Delta}(x)=\psi_{p+1}(x ; h ; m) \sum_{i=0}^{q} \frac{u^{(p+i+1)}(m)}{(p+i+1) !} \Psi_{i}(x ; h ; m) \\
&+\sum_{i=p+q+2}^{\infty} \frac{u^{(i)}(m)}{i !}(x-m)^{i}+\left(v^{\prime}\left(\Gamma_{1}\right)-u^{\prime}\left(\Gamma_{1}\right)\right) L_{1}(x) \\
&+\left(v^{\prime}\left(\Gamma_{p+1}\right)-u^{\prime}\left(\Gamma_{p+1}\right)\right) L_{p+1}(x)+\sum_{i=2}^{p}\left(v\left(\Gamma_{i}\right)-u\left(\Gamma_{i}\right)\right) L_{i}(x),
\end{aligned}
$$

where

$$
\begin{aligned}
\Psi_{i}(x ; h ; m) & =\frac{x^{p+i+1}-\pi^{\Delta} x^{p+i+1}}{\psi_{p+1}(x ; h ; m)} \\
& =\sum_{k=0}^{[i / 2]} a_{i-2 k, i}(x-m)^{i-2 k}, i=0, \ldots, q,
\end{aligned}
$$

with

$$
a_{i, i}=1, a_{i-2 k, i}=-\sum_{l=1}^{\min \left(k,\left[\frac{p+1}{2}\right]\right)} a_{i-2(k-l), i} \hat{a}_{p+1-2 l}^{p+1}, k=1, \ldots,\left[\frac{i}{2}\right] .
$$


Remark. From (8) and (40)-(41) we obtain

(42) $\Psi_{0}(x ; h ; m)=1, \Psi_{1}(x ; h ; m)=x-m, \Psi_{2}(x ; h ; m)=(x-m)^{2}+h^{2} \frac{p(p+1)}{8(2 p-3)}$.

Proof. Consider the expansion

$$
u(x)-U_{I, \Delta}(x)=u(x)-v(x)+v(x)-\pi^{\Delta} v(x)+\pi^{\Delta} v(x)-U_{I, \Delta}(x) .
$$

From (38) it follows that

$$
u(x)-v(x)=\sum_{i=p+q+2}^{\infty} \frac{u^{(i)}(m)}{i !}(x-m)^{i} .
$$

Since $\pi^{\Delta} v(x)$ is a polynomial of degree $p$, Lemma 2 and the definition of $\pi^{\Delta}$ imply that

$$
\begin{aligned}
\pi^{\Delta} v(x)-U_{I, \Delta}(x)= & \left(v^{\prime}\left(\Gamma_{1}\right)-u^{\prime}\left(\Gamma_{1}\right)\right) L_{1}(x)+\left(v^{\prime}\left(\Gamma_{p+1}\right)-u^{\prime}\left(\Gamma_{p+1}\right)\right) L_{p+1}(x) \\
& +\sum_{i=2}^{p}\left(v\left(\Gamma_{i}\right)-u\left(\Gamma_{i}\right)\right) L_{i}(x) .
\end{aligned}
$$

The remainder of the proof follows exactly the proof in [22] for the Lobatto interpolant.

Several a priori estimates can be derived from Theorem 3 [29]. Throughout the remainder of the paper $C$ denotes a generic constant that may depend on the smoothness of $u, \rho, \mu$ and $\kappa$ but not on $h$. In addition we will also need the $H^{1}$ and $H^{2}$ seminorms $|u|_{1}$ and $|u|_{2}$, respectively. Finally $L^{2}, H^{1}$ and $H^{2}$ norms and seminorms over $\Delta$ are denoted by $\|u\|_{i, \Delta}$ and $|u|_{i, \Delta}$ for $i=0,1,2$.

Corollary 4. Under the hypotheses of Theorem 3 with $u \in H^{p+4}(\Delta)$,

$$
\begin{aligned}
& \left\|u-U_{I, \Delta}\right\|_{0, \Delta}^{2}=\left(\frac{(p-1) !}{(2 p-1) !}\right)^{2} \frac{3(2 p-1) h^{2 p+3}}{8(2 p+3)(2 p+1)(2 p-3)(2 p-5)}\left(u^{(p+1)}(m)\right)^{2} \\
& \quad+O\left(h^{2 p+4}\right), \\
& \left|u-U_{I, \Delta}\right|_{1, \Delta}^{2}=\left(\frac{(p-1) !}{(2 p-1) !}\right)^{2} \frac{(2 p-1) h^{2 p+1}}{2(2 p+1)(2 p-3)}\left(u^{(p+1)}(m)\right)^{2}+O\left(h^{2 p+2}\right), \\
& \left|u-U_{I, \Delta}\right|_{2, \Delta}^{2}=\left(\frac{(p-1) !}{(2 p-2) !}\right)^{2} \frac{h^{2 p-1}}{2 p-1}\left(u^{(p+1)}(m)\right)^{2}+O\left(h^{2 p}\right) .
\end{aligned}
$$

Proof. From Theorem 3 with $q=1$ it follows that

$$
\begin{aligned}
u^{(s)}-U_{I, \Delta}^{(s)}= & \psi_{p+1}^{(s)}(x ; h ; m) \frac{u^{(p+1)}(m)}{(p+1) !} \\
& +\left((x-m) \psi_{p+1}(x ; h ; m)\right)^{(s)} \frac{u^{(p+2)}(m)}{(p+2) !} \\
& +\frac{u^{(p+3)}\left(\nu_{s}\right)}{(p+3) !}(x-m)^{p+3-s}+\left(v^{\prime}\left(\Gamma_{1}\right)-u^{\prime}\left(\Gamma_{1}\right)\right) L_{1}^{(s)}(x) \\
& +\left(v^{\prime}\left(\Gamma_{p+1}\right)-u^{\prime}\left(\Gamma_{p+1}\right)\right) L_{p+1}^{(s)}(x)+\sum_{i=2}^{p}\left(v\left(\Gamma_{i}\right)-u\left(\Gamma_{i}\right)\right) L_{i}^{(s)}(x),
\end{aligned}
$$


where $\nu_{s} \in \Delta, s=0,1,2$. From (49) we obtain

$$
\begin{aligned}
& \left\|u^{(s)}-U_{I, \Delta}^{(s)}\right\|_{0, \Delta} \leq \| \psi_{p+1}^{(s)}(x ; h ; m) \frac{u^{(p+1)}(m)}{(p+1) !} \\
& \quad+\left((x-m) \psi_{p+1}(x ; h ; m)\right)^{(s)} \frac{u^{(p+2)}(m)}{(p+2) !} \|_{0, \Delta} \\
& \quad+\frac{\max _{x \in \Delta}\left|u^{(p+3)}(x)\right|}{(p+3) !}\left\|(x-m)^{p+3-s}\right\|_{0, \Delta}+\left|v^{\prime}\left(\Gamma_{1}\right)-u^{\prime}\left(\Gamma_{1}\right)\right|\left\|L_{1}^{(s)}(x)\right\|_{0, \Delta} \\
& \quad+\left|v^{\prime}\left(\Gamma_{p+1}\right)-u^{\prime}\left(\Gamma_{p+1}\right)\right|\left\|L_{p+1}^{(s)}(x)\right\|_{0, \Delta}+\sum_{i=2}^{p}\left|v\left(\Gamma_{i}\right)-u\left(\Gamma_{i}\right)\right|\left\|L_{i}^{(s)}(x)\right\|_{0, \Delta}, \\
& s=0,1,2 .
\end{aligned}
$$

Using (44) yields the bounds

$$
\left|v^{\prime}\left(\Gamma_{i}\right)-u^{\prime}\left(\Gamma_{i}\right)\right| \leq C h^{p+2}, i=1, p+1,\left|v\left(\Gamma_{i}\right)-u\left(\Gamma_{i}\right)\right| \leq C h^{p+3}, i=2, \ldots, p .
$$

A direct computation shows that

$$
\left\|(x-m)^{j}\right\|_{0, \Delta} \leq C h^{j+1 / 2} .
$$

From (34)-(36) it follows that

$$
\begin{gathered}
\left\|L_{i}^{(s)}(x)\right\|_{0, \Delta} \leq C h^{3 / 2-s}, i=1, p+1, \\
\left\|L_{i}^{(s)}(x)\right\|_{0, \Delta} \leq C h^{1 / 2-s}, i=2, \ldots, p, s=0,1,2 .
\end{gathered}
$$

Now

$$
\begin{aligned}
& \left\|\psi_{p+1}^{(s)}(x ; h ; m) \frac{u^{(p+1)}(m)}{(p+1) !}+\left((x-m) \psi_{p+1}(x ; h ; m)\right)^{(s)} \frac{u^{(p+2)}(m)}{(p+2) !}\right\|_{0, \Delta}^{2} \\
& =\left(\frac{u^{(p+1)}(m)}{(p+1) !}\right)^{2}\left\|\psi_{p+1}^{(s)}(x ; h ; m)\right\|_{0, \Delta}^{2} \\
& \quad+\left(\frac{u^{(p+2)}(m)}{(p+2) !}\right)^{2}\left\|\left((x-m) \psi_{p+1}(x ; h ; m)\right)^{(s)}\right\|_{0, \Delta}^{2}, s=0,1,2,
\end{aligned}
$$

since the polynomial $\psi_{p+1}^{(s)}(x ; h ; m)\left((x-m) \psi_{p+1}^{2}(x ; h ; m)\right)^{(s)}$ is odd so that the inner product term in (54) is zero. Applying (14) and (17) to (54) with $s=0$ and the properties of Legendre polynomials gives

$$
\begin{aligned}
& \left\|\psi_{p+1}(x ; h ; m) \frac{u^{(p+1)}(m)}{(p+1) !}+(x-m) \psi_{p+1}(x ; h ; m) \frac{u^{(p+2)}(m)}{(p+2) !}\right\|_{0, \Delta}^{2} \\
& \quad \leq \frac{h^{2 p+3}}{8}\left(\frac{(p-1) !}{(2 p-1) !}\right)^{2} \frac{3(2 p-1)}{(2 p+3)(2 p+1)(2 p-3)(2 p-5)}\left(u^{(p+1)}(m)\right)^{2}+C h^{2 p+5} .
\end{aligned}
$$

Combining (50)-(53) with $s=0$ and (55) produces

$$
\left\|u-U_{I, \Delta}\right\|_{0, \Delta}^{2} \leq\left(\frac{(p-1) !}{(2 p-1) !}\right)^{2} \frac{3(2 p-1) h^{2 p+3}}{8(2 p+3)(2 p+1)(2 p-3)(2 p-5)}\left(u^{(p+1)}(m)\right)^{2}+C h^{2 p+4} .
$$

Similarly we can use (49) with $s=0$ to show that

$$
\left(\frac{(p-1) !}{(2 p-1) !}\right)^{2} \frac{3(2 p-1) h^{2 p+3}}{8(2 p+3)(2 p+1)(2 p-3)(2 p-5)}\left(u^{(p+1)}(m)\right)^{2}-C h^{2 p+4} \leq\left\|u-U_{I, \Delta}\right\|_{0, \Delta}^{2},
$$


and, thus (56)-(57) yield (46).

Using (14), (15), (18) and (54) with $s=1$ and the properties of Legendre polynomials yields

$$
\begin{aligned}
& \left\|\psi_{p+1}^{\prime}(x ; h ; m) \frac{u^{(p+1)}(m)}{(p+1) !}+\left(\psi_{p+1}(x ; h ; m)+(x-m) \psi_{p+1}^{\prime}(x ; h ; m)\right) \frac{u^{(p+2)}(m)}{(p+2) !}\right\|_{0, \Delta}^{2} \\
& \quad \leq \frac{h^{2 p+1}}{2}\left(\frac{(p-1) !}{(2 p-1) !}\right)^{2} \frac{(2 p-1)}{(2 p+1)(2 p-3)}\left(u^{(p+1)}(m)\right)^{2}+C h^{2 p+3} .
\end{aligned}
$$

Combining (50)-(53) with $s=1$ and (58) gives

$$
\left|u-U_{I, \Delta}\right|_{1, \Delta}^{2} \leq\left(\frac{(p-1) !}{(2 p-1) !}\right)^{2} \frac{(2 p-1) h^{2 p+1}}{2(2 p+1)(2 p-3)}\left(u^{(p+1)}(m)\right)^{2}+C h^{2 p+2},
$$

with (47) following equivalently to (46).

Finally from (15), (16), (19) and (54) with $s=2$ and the properties of Legendre polynomials we obtain

$$
\begin{aligned}
& \left\|\psi_{p+1}^{\prime \prime}(x ; h ; m) \frac{u^{(p+1)}(m)}{(p+1) !}+\left(2 \psi_{p+1}^{\prime}(x ; h ; m)+(x-m) \psi_{p+1}^{\prime \prime}(x ; h ; m)\right) \frac{u^{(p+2)}(m)}{(p+2) !}\right\|_{0, \Delta} \\
& \leq \frac{h^{2 p-1}}{(2 p-1)}\left(\frac{(p-1) !}{(2 p-2) !}\right)^{2}\left(u^{(p+1)}(m)\right)^{2}+C h^{2 p} .
\end{aligned}
$$

Then using (50)-(53) with $s=2$ and (60) in an analogous manner produces

$$
\left|u-U_{I, \Delta}\right|_{2, \Delta}^{2} \leq\left(\frac{(p-1) !}{(2 p-2) !}\right)^{2} \frac{h^{2 p-1}}{(2 p-1)}\left(u^{(p+1)}(m)\right)^{2}+O\left(h^{2 p}\right),
$$

and thus, following the earlier results, (48).

The second interpolant approximates $u \in H^{p+4}(\Omega)$. Let

$$
\Delta_{\Omega}:=\left\{a=x_{0}<x_{1}<\cdots<x_{N}=b\right\}
$$

be a partition of $\Omega$ into $N$ subintervals $\Omega_{k}=\left(x_{k-1}, x_{k}\right), k=1, \ldots, N$, and let $S^{\Delta_{\Omega}}$ be the corresponding vector space of piecewise differentiable polynomials whose restriction to $\bar{\Omega}_{k}$ is polynomials of degree $p_{k}$. Additionally let $S^{\Delta_{\Omega},+}$ be the space of piecewise differentiable polynomials whose restriction to $\bar{\Omega}_{k}$ is polynomials of degree $p_{k}+1$. Let $h_{k}=x_{k}-x_{k-1}$ and $m_{k}=\left(x_{k}+x_{k-1}\right) / 2$ be the interval lengths and midpoints of $\Omega_{k}, k=1, \ldots, N$, respectively. In typical $h$ - and $h p$-adaptive codes that use binary refinement [20] the $h_{k}$ satisfy

$$
\frac{h_{k}}{h_{k-1}} \in \mathcal{H} \equiv\left\{\frac{1}{2}, 1,2\right\},
$$

and this assumption will be made throughout. For $p$ - and $h p$-adaptive codes we also require that the $p_{k}$ satisfy the order smoothness condition [20] (see Example $5.1)$

$$
\left|p_{k}-p_{k-1}\right| \leq 1 .
$$

Let $H \equiv h_{\max }=\max _{1 \leq k \leq N} h_{k}, h_{\min }=\min _{1 \leq k \leq N} h_{k}$. In the convergence proofs of sections 3 and 4 the grid $\Delta_{\Omega}$ is refined in such a way that $H \rightarrow 0$ and $\lambda \equiv h_{\max } / h_{\min }$ is kept constant. Thus $H / \lambda \leq h_{k} \leq H, k=1, \ldots, N$. With a slight abuse of notation $\Delta_{\Omega}$ and its associated vector space $S^{\Delta_{\Omega}}$ are referred to as the grid. A grid is said to be spatially uniform if $h_{k}=h, k=1, \ldots, N$. It is of uniform order $p$ if 
$p_{k}=p, k=1, \ldots, N$. In this case the vector space is designated as $S^{\Delta_{\Omega}, p}$. If a grid is both spatially uniform and of uniform order $p$, it is said to be uniform.

Consider an interpolant on $\Delta_{\Omega}, U_{I}(x) \in S^{\Delta_{\Omega}}$ of $u(x)$ having the form

$$
U_{I}(x)=\sum_{k=0}^{N} \sum_{i=2}^{3} \bar{U}_{i, k} \phi_{i, k}(x)+\sum_{k=1}^{N} \sum_{i=4}^{p_{k}} \bar{U}_{i, k} \phi_{i, k}(x) .
$$

The functions

$$
\begin{aligned}
& \phi_{2, k}(x)= \begin{cases}\Phi_{2}\left(x ; h_{k} ; m_{k}\right) & x_{k-1} \leq x \leq x_{k} \\
\Phi_{0}\left(x ; h_{k+1} ; m_{k+1}\right) & x_{k} \leq x \leq x_{k+1}, k=0,1, \ldots, N \\
0 & \text { otherwise }\end{cases} \\
& \phi_{3, k}(x)= \begin{cases}\Phi_{3}\left(x ; h_{k} ; m_{k}\right) & x_{k-1} \leq x \leq x_{k} \\
\Phi_{1}\left(x ; h_{k+1} ; m_{k+1}\right) & x_{k} \leq x \leq x_{k+1}, k=0,1, \ldots, N \\
0 & \text { otherwise }\end{cases}
\end{aligned}
$$

and

$$
\phi_{i, k}(x)=\left\{\begin{array}{ll}
\Phi_{i}\left(x ; h_{k} ; m_{k}\right) & x_{k-1} \leq x<x_{k}, \\
0 & \text { otherwise, }
\end{array}, i=4,5, \ldots, p_{k}, k=1,2, \ldots, N,\right.
$$

comprise a hierarchical basis for $S^{\Delta_{\Omega}}$. As on $\Delta$ the coefficients $\bar{U}_{i, k}$ are defined by requiring

$$
\begin{gathered}
\left.U_{I}\left(x_{i, k}\right)\right|_{\Omega_{k}}=u\left(x_{i, k}\right), i=3, \ldots, p_{k}-1, k=1, \ldots, N, \\
U_{I}^{\prime}\left(x_{i, k}\right)=u^{\prime}\left(x_{i, k}\right), U_{I}\left(x_{i, k}\right)=u\left(x_{i, k}\right), i=1,2, p_{k}, p_{k}+1, k=0, \ldots, N,
\end{gathered}
$$

where $x_{1, k}=x_{2, k}=x_{k-1}, x_{p, k}=x_{p+1, k}=x_{k}$ and $x_{k-1}<x_{i, k}<x_{k} i=3, \ldots$, $p_{k}-1$, are the distinct roots of $\Phi_{p_{k}+1}\left(x ; h_{k} ; m_{k}\right)$. Let $\pi$ be the linear operator that projects functions in $H^{p+4}(\Omega)$ onto the space $S^{\Delta_{\Omega}}$ according to (69)-(70). The a priori estimates of Corollary 4 extend naturally to $\Omega$ [29] as described in the following corollary.

Corollary 5. Let $u \in H^{p+4}(\Omega)$ and let $U_{I} \in S^{\Delta_{\Omega}, p}$ be its Hermite-Lobatto interpolant (65) - (70). Then

$$
\begin{aligned}
& \left\|u-U_{I}\right\|_{2}^{2}=\frac{1}{2 p-1}\left(\frac{(p-1) !}{(2 p-2) !}\right)^{2} \sum_{k=1}^{N} h_{k}^{2 p-1}\left(u^{(p+1)}\left(m_{k}\right)\right)^{2} \\
& +O\left(H^{2 p}\right) \leq C H^{2(p-1)}, \\
& \left\|u-U_{I}\right\|_{s}^{2} \leq C H^{2(p+1-s)}, s=0,1 .
\end{aligned}
$$

Proof. The proof follows directly from Corollary 4.

The Galerkin form of (11)-(2) consists in finding $u \in H_{0}^{2}(\Omega)$ such that

$$
\begin{aligned}
A(u, v) & =(f, v), \forall v \in H_{0}^{2}(\Omega) \\
A(u, v) & =\int_{a}^{b}\left(\rho(x) u^{\prime \prime}(x) v^{\prime \prime}(x)+\mu(x) u^{\prime}(x) v^{\prime}(x)+\kappa(x) u(x) v(x)\right) d x \\
(f, v) & =\int_{a}^{b} f(x) v(x) d x .
\end{aligned}
$$

The bilinear form $A(u, v)$ is both continuous (bounded) and coercive as shown in the next lemma. 
Lemma 6. For $A(u, v)$ defined in (74) with $\rho(x), \mu(x)$ and $\kappa(x)$ satisfying (6), $\exists \alpha, \beta$ such that

$$
\alpha\|u\|_{2}^{2} \leq A(u, u) \leq \beta\|u\|_{2}^{2} .
$$

Proof. The proof is a straightforward extension of the result for second-order equations [29, 31].

The finite element solution $U \in S_{0}^{\Delta_{\Omega}}$ has the same form as $U_{I}$,

$$
U(x)=\sum_{k=0}^{N} \sum_{i=2}^{3} U_{i, k} \phi_{i, k}(x)+\sum_{k=1}^{N} \sum_{i=4}^{p_{k}} U_{i, k} \phi_{i, k}(x),
$$

where the subscript 0 indicates that $U$ satisfies (2). Then $U$ is the solution of

$$
A(U, V)=(f, V), \forall V \in S_{0}^{\Delta_{\Omega}} .
$$

In the next lemma and corollary we present several a priori error estimates and a superconvergence result needed to prove asymptotic equivalence in the subsequent lemma.

Lemma 7. Let $u \in H^{p+4}(\Omega)$ be the solution of (73), $U_{I} \in S_{0}^{\Delta_{\Omega}, p}$ be its HermiteLobatto interpolant (65)-(70) and $U \in S_{0}^{\Delta_{\Omega}, p}$ be the solution of (78). Then

$$
\begin{aligned}
\|u-U\|_{s} & \leq C H^{p+1-s}, s=0,1,2, \\
\left\|U_{I}-U\right\|_{2} & \leq C H^{p} .
\end{aligned}
$$

Proof. [1, 27.

Corollary 8. Under the hypotheses of Lemma 7,

$$
\left\|U_{I}-U\right\|_{s} \leq C H^{p+1-s}, s=0,1 .
$$

Proof. The result follows from Corollary 5 and Lemma 7.

Lemma 9. Let the hypotheses of Lemma 7 hold. If

$$
\left\|u-U_{I}\right\|_{2} \geq C H^{p-1} \text {, }
$$

then

$$
\frac{\|u-U\|_{2}}{\left\|u-U_{I}\right\|_{2}}=1+O(H)
$$

Remarks. Equation (83) is a statement of asymptotic equivalence. Condition (82) is slightly stronger than the more typical saturation assumption [4, 34, but is appropriate in the case of smooth solutions. Corollary 5 and Lemma 9 suggest that a posteriori error estimates can be obtained if $u^{(p+1)}\left(m_{k}\right), k=1, \ldots, N$, can be computed. This is described in section 4 .

Proof. The proof follows directly from (80), (82) and the triangle inequality.

In the time-dependent case (3)-(5), the Galerkin form consists in finding $u \in$ $H_{0}^{2}(\Omega) \times C^{\infty}[0, T]$ such that

$$
\left(u_{t}, v\right)+A(u, v)=(f, v), \forall v \in H_{0}^{2}(\Omega), t \in(0, T] .
$$

The finite element solution $U \in S_{0}^{\Delta_{\Omega}} \times C^{\infty}[0, T]$ has the form

$$
U(x, t)=\sum_{k=0}^{N} \sum_{i=2}^{3} U_{i, k}(t) \phi_{i, k}(x)+\sum_{k=1}^{N} \sum_{i=4}^{p_{k}} U_{i, k}(t) \phi_{i, k}(x)
$$


and is the solution of

$$
\left(U_{t}, V\right)+A(U, V)=(f, V), \forall V \in S_{0}^{\Delta_{\Omega}}, t \in(0, T], U(x, 0)=\pi u_{0}(x) .
$$

In the remainder of this section, $U_{I} \in S_{0}^{\Delta_{\Omega}} \times C^{\infty}[0, T]$ corresponds to the HermiteLobatto interpolant computed according to (65)-(70) for each fixed time $t$.

The next several lemmas and corollary describe a priori error estimates and asymptotic convergence in the time-dependent case.

Lemma 10. Let $u \in H^{p+4}(\Omega)$ be the solution of (4ㅁ $), U_{I} \in S_{0}^{\Delta_{\Omega}, p} \times C^{\infty}[0, T]$ its Hermite-Lobatto interpolant (65)-(70) for each fixed time $t$ and $U \in S_{0}^{\Delta_{\Omega}, p} \times$ $C^{\infty}[0, T]$ be the solution of (86). Then

$$
\begin{aligned}
\left\|u_{t}-U_{t}\right\|_{0} & \leq C H^{p+1} \\
\|u-U\|_{s} & \leq C H^{p+1-s}, s=0,1,2, \\
\left\|U_{I}-U\right\|_{2} & \leq C H^{p} .
\end{aligned}
$$

Before proving Lemma 10 we need the following two lemmas.

Lemma 11. Let $u \in H^{p+4}(\Omega)$ and let $U_{I} \in S^{\Delta_{\Omega}, p}$ be its Hermite-Lobatto interpolant (65)-(70) for each fixed time $t$. Then

$$
\left|a\left(u-U_{I}, W\right)\right| \leq C H^{p}\|u\|_{p+2}\|W\|_{2}, \forall W \in S_{0}^{\Delta_{\Omega}, p} .
$$

Proof. [1].

Lemma 12. Let $\hat{U} \in S_{0}^{\Delta_{\Omega}, p}$, and let the elliptic projection of $u$ satisfy

$$
A(\hat{U}, V)=A(u, V), \forall V \in S_{0}^{\Delta_{\Omega}, p},
$$

where $u \in H^{p+4}(\Omega)$ is the solution of (84) and $U_{I} \in S^{\Delta_{\Omega}, p}$ its Hermite-Lobatto interpolant (65) -(70) for each fixed time $t$. Then

$$
\begin{aligned}
\left\|u_{t}-\hat{U}_{t}\right\|_{0} & \leq C H^{p+1} \\
\|u-\hat{U}\|_{s} & \leq C H^{p+1-s}, s=0,1,2, \\
\left\|U_{I}-\hat{U}\right\|_{2} & \leq C H^{p} .
\end{aligned}
$$

Proof. Following [35] for second-order equations, differentiate (91) with respect to $t$ and use the proof of (79) with $s=0$ to obtain (92). The proof of (93), $s=0,1,2$, follows the proof of (79), $s=0,1,2$, in [27]. Equation (94) is obtained from Lemma 11 following the proof of (80) in [1] by choosing $W=\hat{U}-U_{I}$.

Proof of Lemma 10. The proofs of (87) and (89) follow the approach in [35. From (84) and (86) it follows that

$$
\left(u_{t}-U_{t}, V\right)+A(u-U, V)=0, \forall V \in S_{0}^{\Delta_{\Omega}, p}, t \in(0, T] .
$$

Equations (91) and (95) imply

$$
\left(\hat{U}_{t}-U_{t}, V\right)+A(\hat{U}-U, V)=\left(\hat{U}_{t}-u_{t}, V\right), \forall V \in S_{0}^{\Delta_{\Omega}, p}, t \in(0, T] .
$$

Letting $V=\hat{U}_{t}-U_{t}$ in (96) and using the Cauchy-Schwarz inequality yields

$$
\left\|\hat{U}_{t}-U_{t}\right\|_{0}^{2}+\frac{1}{2} \frac{d}{d t} A(\hat{U}-U, \hat{U}-U) \leq\left\|\hat{U}_{t}-u_{t}\right\|_{0}^{2}+\left\|\hat{U}_{t}-U_{t}\right\|_{0}^{2},
$$

and thus

$$
\frac{1}{2} \frac{d}{d t} A(\hat{U}-U, \hat{U}-U) \leq\left\|\hat{U}_{t}-u_{t}\right\|_{0}^{2}
$$


Integrating (98) over $[0, t]$ results in

$$
\begin{aligned}
A(\hat{U}(\cdot, t)-U(\cdot, t), \hat{U}(\cdot, t)-U(\cdot, t)) & \leq A(\hat{U}(\cdot, 0)-U(\cdot, 0), \hat{U}(\cdot, 0)-U(\cdot, 0)) \\
& +C \int_{0}^{t}\left\|\hat{U}_{t}(\cdot, s)-u_{t}(\cdot, s)\right\|_{0}^{2} d s
\end{aligned}
$$

or applying (176) and (92),

$$
\alpha\|\hat{U}(t)-U(t)\|_{2}^{2} \leq C\|\hat{U}(0)-U(0)\|_{2}^{2}+C H^{2(p+1)} .
$$

Since $U(0)=U_{I}(0)$, (94) implies that

$$
\|\hat{U}(0)-U(0)\|_{2}^{2} \leq C H^{2 p},
$$

and (89) follows from (94), (100) and (101).

From (98) we obtain

$$
\frac{1}{2} \frac{d}{d t} A(\hat{U}-U, \hat{U}-U) \leq C H^{2(p+1)},
$$

where we have used (92). Then (96) with $V=\hat{U}_{t}-U_{t}$ also implies that

$$
\left\|\hat{U}_{t}-U_{t}\right\|_{0}^{2}+\frac{1}{2} \frac{d}{d t} A(\hat{U}-U, \hat{U}-U) \leq \frac{1}{2}\left\|\hat{U}_{t}-u_{t}\right\|_{0}^{2}+\frac{1}{2}\left\|\hat{U}_{t}-U_{t}\right\|_{0}^{2} .
$$

Therefore (92), (102) and (103) imply that

$$
\left\|\hat{U}_{t}-U_{t}\right\|_{0} \leq C H^{p+1},
$$

which yields (87) by using (92) and the triangle inequality.

We obtain (88) with $s=2$ from (89), (93) with $s=2$, (94) and the triangle inequality. The proofs of (88) with $s=0,1$, follow closely the proofs in [15, 27] in the time-independent case using the Aubin-Nitsche technique. Let $w \in H_{0}^{s}(\Omega)$ and let $u_{w} \in H^{p+4}(\Omega) \times H_{0}^{2}(\Omega)$ be the solution of

$$
A\left(v, u_{w}\right)=\langle w, v\rangle_{s}, \forall v \in H_{0}^{2}(\Omega), s=0,1,
$$

where

$$
\langle w, v\rangle_{s}=\int_{a}^{b} w^{(s)}(x) v^{(s)}(x) d x .
$$

Then [15, 27] imply that

$$
\left\|u_{w}\right\|_{4-s} \leq C|w|_{s},\left\|u_{w}-\pi u_{w}\right\|_{2} \leq C H^{2-s}\left\|u_{w}\right\|_{4-s} .
$$

If for each fixed time $t, e=u-U$, then

$$
|e|_{s}=\sup _{w \in H_{0}^{s}(\Omega)} \frac{\langle w, e\rangle_{s}}{|w|_{s}}=\sup _{w \in H_{0}^{s}(\Omega)} \frac{A\left(e, u_{w}\right)}{|w|_{s}} .
$$

The Cauchy-Schwarz inequality together with (87), (95) and (107) imply that

$$
\begin{aligned}
A\left(e, u_{w}\right) & =A\left(e, u_{w}-\pi u_{w}\right)-\left(e_{t}, \pi u_{w}\right) \\
& \leq C\left(\|e\|_{2}\left\|u_{w}-\pi u_{w}\right\|_{2}+\left\|e_{t}\right\|_{0}\left\|\pi u_{w}\right\|_{0}\right) \\
& \leq C\left(H^{p-1}\left\|u_{w}-\pi u_{w}\right\|_{2}+H^{p+1}\left(\left\|u_{w}\right\|_{0}+\left\|\pi u_{w}-u_{w}\right\|_{0}\right)\right. \\
& \leq C\left(H^{p+1-s}\left\|u_{w}\right\|_{4-s}+C H^{p+1}\left(\left\|u_{w}\right\|_{4-s}+H^{2-s}\left\|u_{w}\right\|_{4-s}\right)\right) \\
& \leq C H^{p+1-s}|w|_{s} .
\end{aligned}
$$

Equations (88) with $s=0,1$ are obtained from (109) and (108) with $s=0$ and $s=1$, respectively. 
Corollary 13. Under the hypotheses of Lemma 10,

$$
\left\|U_{I}-U\right\|_{s} \leq C H^{p+1-s}, s=0,1 .
$$

Proof. The result follows from Corollary 5 and Lemma 10.

The next lemma proves asymptotic equivalence in the time-dependent case.

Lemma 14. Let the hypotheses of Lemma 10 hold. If

$$
\left\|u-U_{I}\right\|_{2} \geq C H^{p-1},
$$

then

$$
\frac{\|u-U\|_{2}}{\left\|u-U_{I}\right\|_{2}}=1+O(H) .
$$

Proof. The proof follows directly from (111), Lemma 10 and the triangle inequality.

\section{A posteriori ERror Estimates}

A posteriori error estimates are essential for guiding the adaptive strategies. In this section we present estimators for orders $p_{k}$ and $p_{k}+1$. In particular for the latter case we estimate the error in the solution of (78) or (86) that lies in $S_{0}^{\Delta_{\Omega},+}$, referred to as $U^{+}$. Since the Hermite-Lobatto interpolant is asymptotically equivalent to the finite element solution (Lemmas 9 and 14, respectively) these estimators can be generated by finding approximations of $u^{\left(p_{k}+1\right)}\left(m_{k}\right)$ and $u^{\left(p_{k}+2\right)}\left(m_{k}\right)$. To that end in the steady-state case (78) let

$$
\left.U^{p_{k}+1}(x)\right|_{\Omega_{k}}=U(x)+W_{p_{k}+1, k} \psi_{p_{k}+1, k}(x),
$$

where for notational convenience, $\psi_{p_{k}+1, k}(x)=\psi_{p_{k}+1}\left(x ; h_{k} ; m_{k}\right)$. The coefficient $W_{p_{k}+1, k}$ is determined by requiring that

$$
\begin{aligned}
\hat{A}\left(U^{p_{k}+1}, \psi_{p_{k}+1, k}\right)_{\Omega_{k}}= & \left(f, \psi_{p_{k}+1, k}\right)_{\Omega_{k}} \\
& -\left(\mu U^{\prime}, \psi_{p_{k}+1, k}^{\prime}\right)_{\Omega_{k}}-\left(\kappa U, \psi_{p_{k}+1, k}\right)_{\Omega_{k}}, k=1, \ldots, N
\end{aligned}
$$

where

$$
\hat{A}(u, v)_{\Omega_{k}}=\int_{x_{k-1}}^{x_{k}} \rho(x) u^{\prime \prime}(x) v^{\prime \prime}(x) d x,
$$

and the subscript $\Omega_{k}$ refers to integration over the element $\Omega_{k}$. To estimate the error in $U^{+}$, a second solution,

$$
\left.U^{p_{k}+2}(x)\right|_{\Omega_{k}}=U^{p_{k}+1}(x)+W_{p_{k}+2, k} \psi_{p_{k}+2, k}(x),
$$

is similarly constructed by requiring that

$$
\begin{aligned}
\hat{A}\left(U^{p_{k}+2}, \psi_{p_{k}+2, k}\right)_{\Omega_{k}}= & \left(f, \psi_{p_{k}+2, k}\right)_{\Omega_{k}}-\left(\mu U^{\prime}, \psi_{p_{k}+2, k}^{\prime}\right)_{\Omega_{k}} \\
& -\left(\kappa U, \psi_{p_{k}+2, k}\right)_{\Omega_{k}}, k=1, \ldots, N .
\end{aligned}
$$

For the time-dependent case (86) the equations are

$$
\begin{aligned}
\hat{A}\left(U^{p_{k}+1}, \psi_{p_{k}+1, k}\right)_{\Omega_{k}}= & \left(f, \psi_{p_{k}+1, k}\right)_{\Omega_{k}}-\left(\mu U^{\prime}, \psi_{p_{k}+1, k}^{\prime}\right)_{\Omega_{k}} \\
& -\left(\kappa U, \psi_{p_{k}+1, k}\right)_{\Omega_{k}}-\left(U_{t}, \psi_{p_{k}+1, k}\right)_{\Omega_{k}}, \\
\hat{A}\left(U^{p_{k}+2}, \psi_{p_{k}+2, k}\right)_{\Omega_{k}}= & \left(f, \psi_{p_{k}+2, k}\right)_{\Omega_{k}}-\left(\mu U^{\prime}, \psi_{p_{k}+2, k}^{\prime}\right)_{\Omega_{k}} \\
& -\left(\kappa U, \psi_{p_{k}+2, k}\right)_{\Omega_{k}}-\left(U_{t}, \psi_{p_{k}+2, k}\right)_{\Omega_{k}},
\end{aligned}
$$


$k=1, \ldots, N$. We will show that on grids with uniform order $p, W_{p+1, k}=$ $\frac{u^{(p+1)}\left(m_{k}\right)}{(p+1) !}+O\left(H^{3 / 2}\right)$ and $W_{p+2, k}=\frac{u^{(p+2)}\left(m_{k}\right)}{(p+2) !}+O\left(H^{1 / 2}\right)$.

To do so we need two additional interpolants $U_{I}^{p+1}$ and $U_{I}^{p+2}$ defined by

$$
\begin{aligned}
& \left.U_{I}^{p+1}(x)\right|_{\Omega_{k}}=U_{I}(x)+\bar{W}_{p+1, k} \psi_{p+1, k}(x), \\
& \left.U_{I}^{p+2}(x)\right|_{\Omega_{k}}=U_{I}^{p+1}(x)+\bar{W}_{p+2, k} \psi_{p+2, k}(x),
\end{aligned}
$$

where $\bar{W}_{p+1, k}$ and $\bar{W}_{p+2, k}$ are determined by requiring that

$$
\hat{A}\left(u-U_{I}^{p+1}, \psi_{p+1, k}\right)_{\Omega_{k}}=0, \hat{A}\left(u-U_{I}^{p+2}, \psi_{p+2, k}\right)_{\Omega_{k}}=0, k=1, \ldots, N,
$$

respectively.

In the remainder of the paper $\nu_{k}$ denotes the point in $\Omega_{k}$ obtained in truncating the series in Taylor's Theorem.

Theorem 15. Let $u \in H_{0}^{p+4}(\Omega)$ be the solution of (73) and let $U_{I}^{p+1} \in S_{0}^{\Delta_{\Omega}, p+1}$ be defined by (120)-(121). Then

$$
\bar{W}_{p+1, k}=\frac{u^{(p+1)}\left(m_{k}\right)}{(p+1) !}+O\left(h_{k}^{2}\right), k=1, \ldots, N .
$$

Proof. From (120) and Theorem 3 with $q=1$ we obtain on $\Omega_{k}$,

$$
\begin{aligned}
\left(u-U_{I}^{p+1}\right)^{\prime \prime}=\left(u-U_{I}+U_{I}-U_{I}^{p+1}\right)^{\prime \prime} & \\
= & \left(\frac{u^{(p+1)}\left(m_{k}\right)}{(p+1) !}-\bar{W}_{p+1, k}\right) \psi_{p+1, k}^{\prime \prime}(x) \\
& +\frac{u^{(p+2)}\left(m_{k}\right)}{(p+2) !}\left(\left(x-m_{k}\right) \psi_{p+1, k}(x)\right)^{\prime \prime}+u^{(p+3)}\left(\nu_{k}\right) \frac{\left(x-m_{k}\right)^{p+1}}{(p+1) !} \\
& +\left(v^{\prime}\left(x_{1, k}\right)-u^{\prime}\left(x_{1, k}\right)\right) L_{1}^{\prime \prime}(x)+\left(v^{\prime}\left(x_{p+1, k}\right)-u^{\prime}\left(x_{p+1, k}\right)\right) L_{p+1}^{\prime \prime}(x) \\
& +\sum_{i=2}^{p}\left(v\left(x_{i, k}\right)-u\left(x_{i, k}\right)\right) L_{i}^{\prime \prime}(x) .
\end{aligned}
$$

Substituting (123) into (120) gives

$$
\begin{aligned}
& \left|\frac{u^{(p+1)}\left(m_{k}\right)}{(p+1) !}-\bar{W}_{p+1, k}\right| \times \int_{x_{k-1}}^{x_{k}} \rho(x)\left(\psi_{p+1, k}^{\prime \prime}(x)\right)^{2} d x \\
& \quad \leq\left|\frac{u^{(p+2)}\left(m_{k}\right)}{(p+2) !}\right|\left|\int_{x_{k-1}}^{x_{k}} \rho(x)\left(\left(x-m_{k}\right) \psi_{p+1, k}(x)\right)^{\prime \prime} \psi_{p+1, k}^{\prime \prime}(x) d x\right| \\
& \quad+\frac{1}{(p+1) !}\left|\int_{x_{k-1}}^{x_{k}} \rho(x) u^{(p+3)}\left(\nu_{k}\right)\left(x-m_{k}\right)^{p+1} \psi_{p+1, k}^{\prime \prime}(x) d x\right| \\
& \quad+\left|v^{\prime}\left(x_{1, k}\right)-u^{\prime}\left(x_{1, k}\right)\right|\left|\int_{x_{k-1}}^{x_{k}} \rho(x) L_{1}^{\prime \prime}(x) \psi_{p+1, k}^{\prime \prime}(x) d x\right| \\
& \quad+\left|v^{\prime}\left(x_{p+1, k}\right)-u^{\prime}\left(x_{p+1, k}\right)\right|\left|\int_{x_{k-1}}^{x_{k}} \rho(x) L_{p+1}^{\prime \prime}(x) \psi_{p+1, k}^{\prime \prime}(x) d x\right| \\
& \quad+\sum_{i=2}^{p}\left|v\left(x_{i, k}\right)-u\left(x_{i, k}\right)\right|\left|\int_{x_{k-1}}^{x_{k}} \rho(x) L_{i}^{\prime \prime}(x) \psi_{p+1, k}^{\prime \prime}(x) d x\right| .
\end{aligned}
$$


The following bounds must be computed for all terms in (124). Throughout we rely on the smoothness of $\rho(x)$ and $u(x)$. Using (19) and (21) in the term on the left yields

$$
\begin{aligned}
\int_{x_{k-1}}^{x_{k}} & \rho(x)\left(\psi_{p+1, k}^{\prime \prime}(x)\right)^{2} d x \geq \rho\left(m_{k}\right) \int_{x_{k-1}}^{x_{k}}\left(\psi_{p+1, k}^{\prime \prime}(x)\right)^{2} d x \\
& -\left|\rho^{\prime}\left(m_{k}\right)\right|\left|\int_{x_{k-1}}^{x_{k}}\left(x-m_{k}\right)\left(\psi_{p+1, k}^{\prime \prime}(x)\right)^{2} d x\right| \\
& -\frac{1}{2}\left|\int_{x_{k-1}}^{x_{k}} \rho^{\prime \prime}\left(\nu_{k}\right)\left(x-m_{k}\right)^{2}\left(\psi_{p+1, k}^{\prime \prime}(x)\right)^{2} d x\right| \\
\geq & \rho\left(m_{k}\right) \frac{h_{k}^{2 p-1}}{2 p-1}\left(\frac{(p+1) !(p-1) !}{(2 p-2) !}\right)^{2}\left(1-C h_{k}^{2}\right) \geq 0,
\end{aligned}
$$

for $h_{k}$ sufficiently small, where the middle integral on the right is zero since the integrand is an odd function on $\Omega_{k}$.

The first term on the right of (124) satisfies

$$
\begin{aligned}
& \left|\frac{u^{(p+2)}\left(m_{k}\right)}{(p+2) !}\right| \times\left|\int_{x_{k-1}}^{x_{k}} \rho(x)\left(\left(x-m_{k}\right) \psi_{p+1, k}(x)\right)^{\prime \prime} \psi_{p+1, k}^{\prime \prime}(x) d x\right| \\
& \leq C\left(\rho\left(m_{k}\right)\left|\int_{x_{k-1}}^{x_{k}}\left(\left(x-m_{k}\right) \psi_{p+1, k}(x)\right)^{\prime \prime} \psi_{p+1, k}^{\prime \prime}(x) d x\right|\right. \\
& \left.+\left|\int_{x_{k-1}}^{x_{k}} \rho^{\prime}\left(\nu_{k}\right)\left(x-m_{k}\right)\left(\left(x-m_{k}\right) \psi_{p+1, k}(x)\right)^{\prime \prime} \psi_{p+1, k}^{\prime \prime}(x) d x\right|\right) \leq C h_{k}^{2 p+1},
\end{aligned}
$$

where we have used (21), and the first integral on the right of (126) vanishes since the integrand is an odd function on $\Omega_{k}$. For the second term we have from (21) that

$$
\frac{1}{(p+1) !}\left|\int_{x_{k-1}}^{x_{k}} \rho(x) u^{(p+3)}\left(\nu_{k}\right)\left(x-m_{k}\right)^{p+1} \psi_{p+1, k}^{\prime \prime}(x) d x\right| \leq C h_{k}^{2 p+1} .
$$

Since $\left|L_{i}^{\prime \prime}(x)\right| \leq C h_{k}^{-1}, i=1, p+1$, the next two terms yield

$$
\left|v^{\prime}\left(x_{i, k}\right)-u^{\prime}\left(x_{i, k}\right)\right|\left|\int_{x_{k-1}}^{x_{k}} \rho(x) L_{i}^{\prime \prime}(x) \psi_{p+1, k}^{\prime \prime}(x) d x\right| \leq C h_{k}^{2 p+1}, i=1, p+1,
$$

where we have again used (21) together with (51). Finally

$$
\left|v\left(x_{i, k}\right)-u\left(x_{i, k}\right)\right|\left|\int_{x_{k-1}}^{x_{k}} \rho(x) L_{i}^{\prime \prime}(x) \psi_{p+1, k}^{\prime \prime}(x) d x\right| \leq C h_{k}^{2 p+1}, i=2, \ldots, p,
$$

using (51) and $\left|L_{i}^{\prime \prime}(x)\right| \leq C h_{k}^{-2}, i=2, \ldots, p$. Combining (124)-(129) we obtain the result. 
Theorem 16. Let $u \in H_{0}^{p+5}(\Omega)$ be the solution of (73) and let $U_{I}^{p+2} \in S_{0}^{\Delta_{\Omega}, p+2}$ be defined by (120)-(121). Then

$$
\bar{W}_{p+2, k}=\frac{u^{(p+2)}\left(m_{k}\right)}{(p+2) !}+O\left(h_{k}^{2}\right), k=1, \ldots, N .
$$

Proof. From (20), (120) and Theorem 3 with $q=2$ on $\Omega_{k}$ it follows that

$$
\begin{aligned}
\left(u-U_{I}^{p+2}\right)^{\prime \prime}=\left(u-U_{I}+U_{I}-U_{I}^{p+1}+U_{I}^{p+1}-U_{I}^{p+2}\right)^{\prime \prime} \\
=\left(\frac{u^{(p+1)}\left(m_{k}\right)}{(p+1) !}-\bar{W}_{p+1, k}\right) \psi_{p+1, k}^{\prime \prime}(x) \\
\quad+\left(\frac{u^{(p+2)}\left(m_{k}\right)}{(p+2) !}-\bar{W}_{p+2, k}\right) \psi_{p+2, k}^{\prime \prime}(x) \\
\quad+\frac{u^{(p+2)}\left(m_{k}\right)}{(p+2) !} \frac{h_{k}^{2}}{4} \frac{(p+1)(p-3)}{(2 p-1)(2 p-3)} \psi_{p, k}^{\prime \prime}(x) \\
\quad+u^{(p+3)}\left(m_{k}\right) \frac{\left(x-m_{k}\right)^{p+1}}{(p+1) !}+u^{(p+4)}\left(\nu_{k}\right) \frac{\left(x-m_{k}\right)^{p+2}}{(p+2) !} \\
\quad+\left(v^{\prime}\left(x_{1, k}\right)-u^{\prime}\left(x_{1, k}\right)\right) L_{1}^{\prime \prime}(x)+\left(v^{\prime}\left(x_{p+1, k}\right)-u^{\prime}\left(x_{p+1, k}\right)\right) L_{p+1}^{\prime \prime}(x) \\
\quad+\sum_{i=2}^{p}\left(v\left(x_{i, k}\right)-u\left(x_{i, k}\right)\right) L_{i}^{\prime \prime}(x) .
\end{aligned}
$$

Substituting (131) into (120) results in

$$
\begin{aligned}
& \left|\frac{u^{(p+2)}\left(m_{k}\right)}{(p+2) !}-\bar{W}_{p+2, k}\right| \times \int_{x_{k-1}}^{x_{k}} \rho(x)\left(\psi_{p+2, k}^{\prime \prime}(x)\right)^{2} d x \\
& \leq\left|\frac{u^{(p+1)}\left(m_{k}\right)}{(p+1) !}-\bar{W}_{p+1, k}\right|\left|\int_{x_{k-1}}^{x_{k}} \rho(x) \psi_{p+1, k}^{\prime \prime}(x) \psi_{p+2, k}^{\prime \prime}(x) d x\right| \\
& \quad+\left|\frac{u^{(p+2)}\left(m_{k}\right)}{(p+2) !}\right| \frac{h_{k}^{2}}{4} \frac{(p+1)(p-3)}{(2 p-1)(2 p-3)}\left|\int_{x_{k-1}}^{x_{k}} \rho(x) \psi_{p, k}^{\prime \prime}(x) \psi_{p+2, k}^{\prime \prime}(x) d x\right| \\
& \quad+\left|\frac{u^{(p+3)}\left(m_{k}\right)}{(p+1) !}\right|\left|\int_{x_{k-1}}^{x_{k}} \rho(x)\left(x-m_{k}\right)^{p+1} \psi_{p+2, k}^{\prime \prime}(x) d x\right| \\
& \quad+\frac{1}{(p+2) !}\left|\int_{x_{k-1}}^{x_{k}} \rho(x) u^{(p+4)}\left(\nu_{k}\right)\left(x-m_{k}\right)^{p+2} \psi_{p+2, k}^{\prime \prime}(x) d x\right| \\
& \quad+\left|v^{\prime}\left(x_{1, k}\right)-u^{\prime}\left(x_{1, k}\right)\right|\left|\int_{x_{k-1}}^{x_{k}} \rho(x) L_{1}^{\prime \prime}(x) \psi_{p+2, k}^{\prime \prime}(x) d x\right| \\
& \quad+\left|v^{\prime}\left(x_{p+1, k}\right)-u^{\prime}\left(x_{p+1, k}\right)\right|\left|\int_{x_{k-1}}^{x_{k}} \rho(x) L_{p+1}^{\prime \prime}(x) \psi_{p+2, k}^{\prime \prime}(x) d x\right| \\
& \quad+\sum_{i=2}^{p}\left|v\left(x_{i, k}\right)-u\left(x_{i, k}\right)\right|\left|\int_{x_{k-1}}^{x_{k}} \rho(x) L_{i}^{\prime \prime}(x) \psi_{p+2, k}^{\prime \prime}(x) d x\right| .
\end{aligned}
$$


Again bounds must be computed for all terms in (132) and we use the smoothness of $\rho(x)$ and $u(x)$ throughout. Using equations (19) and (21) in the term on the left gives

$$
\begin{aligned}
\int_{x_{k-1}}^{x_{k}} & \rho(x)\left(\psi_{p+2, k}^{\prime \prime}(x)\right)^{2} d x \geq \rho\left(m_{k}\right) \int_{x_{k-1}}^{x_{k}}\left(\psi_{p+2, k}^{\prime \prime}(x)\right)^{2} d x \\
& -\left|\rho^{\prime}\left(m_{k}\right)\right|\left|\int_{x_{k-1}}^{x_{k}}\left(x-m_{k}\right)\left(\psi_{p+2, k}^{\prime \prime}(x)\right)^{2} d x\right| \\
& -\frac{1}{2}\left|\int_{x_{k-1}}^{x_{k}} \rho\left(\nu_{k}\right)\left(x-m_{k}\right)^{2}\left(\psi_{p+2, k}^{\prime \prime}(x)\right)^{2} d x\right| \\
\geq & \rho\left(m_{k}\right) \frac{h_{k}^{2 p+1}}{2 p+1}\left(\frac{(p+2) ! p !}{(2 p) !}\right)^{2}\left(1-C h_{k}^{2}\right) \geq 0,
\end{aligned}
$$

for $h_{k}$ sufficiently small, where as before the middle integral on the right is zero since the integrand is an odd function on $\Omega_{k}$.

Theorem 15 and (21) imply that the first term on the right of (132) satisfies

$$
\begin{aligned}
& \left|\frac{u^{(p+1)}\left(m_{k}\right)}{(p+1) !}-\bar{W}_{p+1, k}\right| \times\left|\int_{x_{k-1}}^{x_{k}} \rho(x) \psi_{p+1, k}^{\prime \prime}(x) \psi_{p+2, k}^{\prime \prime}(x) d x\right| \\
& \leq C h_{k}^{2}\left(\rho\left(m_{k}\right)\left|\int_{x_{k-1}}^{x_{k}} \psi_{p+1, k}^{\prime \prime}(x) \psi_{p+2, k}^{\prime \prime}(x) d x\right|\right. \\
& \left.\quad+\left|\int_{x_{k-1}}^{x_{k}} \rho^{\prime}\left(\nu_{k}\right)\left(x-m_{k}\right) \psi_{p+1, k}^{\prime \prime}(x) \psi_{p+2, k}^{\prime \prime}(x) d x\right|\right) \\
& \leq C h_{k}^{2 p+3}
\end{aligned}
$$

since the first integral on the right is zero. The second term on the right is bounded by

$$
\begin{aligned}
& \left|\frac{u^{(p+2)}\left(m_{k}\right)}{(p+2) !}\right| \times \frac{h_{k}^{2}}{4} \frac{(p+1)(p-3)}{(2 p-1)(2 p-3)}\left|\int_{x_{k-1}}^{x_{k}} \rho(x) \psi_{p, k}^{\prime \prime}(x) \psi_{p+2, k}^{\prime \prime}(x) d x\right| \\
& \leq C h_{k}^{2}\left(\rho\left(m_{k}\right)\left|\int_{x_{k-1}}^{x_{k}} \psi_{p, k}^{\prime \prime}(x) \psi_{p+2, k}^{\prime \prime}(x) d x\right|\right. \\
& +\left|\rho^{\prime}\left(m_{k}\right)\right|\left|\int_{x_{k-1}}^{x_{k}}\left(x-m_{k}\right) \psi_{p, k}^{\prime \prime}(x) \psi_{p+2, k}^{\prime \prime}(x) d x\right| \\
& \left.+\frac{1}{2}\left|\int_{x_{k-1}}^{x_{k}} \rho^{\prime \prime}\left(\nu_{k}\right)\left(x-m_{k}\right)^{2} \psi_{p, k}^{\prime \prime}(x) \psi_{p+2, k}^{\prime \prime}(x) d x\right|\right) \leq C h_{k}^{2 p+3},
\end{aligned}
$$


using (21) since the first two integrals on the right are zero due to the orthogonality of the Legendre polynomials. For the third integral we obtain

$$
\begin{aligned}
& \left|\frac{u^{(p+3)}\left(m_{k}\right)}{(p+1) !}\right| \times\left|\int_{x_{k-1}}^{x_{k}} \rho(x)\left(x-m_{k}\right)^{p+1} \psi_{p+2, k}^{\prime \prime}(x) d x\right| \\
& \leq C\left(\rho\left(m_{k}\right)\left|\int_{x_{k-1}}^{x_{k}}\left(x-m_{k}\right)^{p+1} \psi_{p+2, k}^{\prime \prime}(x) d x\right|\right. \\
& \left.\quad+\left|\int_{x_{k-1}}^{x_{k}} \rho^{\prime}\left(\nu_{k}\right)\left(x-m_{k}\right)^{p+2} \psi_{p+2, k}^{\prime \prime}(x) d x\right|\right) \\
& \leq C h_{k}^{2 p+3},
\end{aligned}
$$

where the first integral on the right is zero since the integrand is an odd function on $\Omega_{k}$ and we have used (21). The next integral yields, using (21),

$$
\frac{1}{(p+2) !}\left|\int_{x_{k-1}}^{x_{k}} \rho(x) u^{(p+4)}\left(\nu_{k}\right)\left(x-m_{k}\right)^{p+2} \psi_{p+2, k}^{\prime \prime}(x) d x\right| \leq C h_{k}^{2 p+3} .
$$

The next two integrals satisfy

$$
\begin{aligned}
& \left|v^{\prime}\left(x_{i, k}\right)-u^{\prime}\left(x_{i, k}\right)\right| \times\left|\int_{x_{k-1}}^{x_{k}} \rho(x) L_{1}^{\prime \prime}(x) \psi_{p+2, k}^{\prime \prime}(x) d x\right| \\
& \leq C h_{k}^{p+2}\left(\rho\left(m_{k}\right)\left|\int_{x_{k-1}}^{x_{k}} L_{i}^{\prime \prime}(x) \psi_{p+2, k}^{\prime \prime}(x) d x\right|\right. \\
& \left.\quad+\left|\int_{x_{k-1}}^{x_{k}} \rho^{\prime}\left(\nu_{k}\right)\left(x-m_{k}\right) L_{i}^{\prime \prime}(x) \psi_{p+2, k}^{\prime \prime}(x) d x\right|\right) \\
& \leq C h_{k}^{2 p+3},
\end{aligned}
$$

for $i=1, p+1$ using (21), (51) and $\left|L_{i}^{\prime \prime}(x)\right| \leq C h_{k}^{-1}, i=1, p+1$. Finally using (21), (51) and $\left|L_{i}^{\prime \prime}(x)\right| \leq C h_{k}^{-2}, i=2, \ldots, p$, each term in the sum is bounded by

$$
\begin{aligned}
& \left|v\left(x_{i, k}\right)-u\left(x_{i, k}\right)\right| \times\left|\int_{x_{k-1}}^{x_{k}} \rho(x) L_{i}^{\prime \prime}(x) \psi_{p+2, k}^{\prime \prime}(x) d x\right| \\
& \leq C h_{k}^{p+2}\left(\rho\left(m_{k}\right)\left|\int_{x_{k-1}}^{x_{k}} L_{i}^{\prime \prime}(x) \psi_{p+2, k}^{\prime \prime}(x) d x\right|\right. \\
& \left.\quad+\left|\int_{x_{k-1}}^{x_{k}} \rho^{\prime}\left(\nu_{k}\right)\left(x-m_{k}\right) L_{i}^{\prime \prime}(x) \psi_{p+2, k}^{\prime \prime}(x) d x\right|\right) \\
& \leq C h_{k}^{2 p+3}, i=2, \ldots, p,
\end{aligned}
$$

where the first integral on the right side vanishes from the orthogonality of Legendre polynomials. Combining (132)-(139) yields the result.

We are now in a position to prove the main results. 
Theorem 17. Let $u \in H_{0}^{p+4}(\Omega)$ be the solution of (73) and let $U^{p+1} \in S_{0}^{\Delta_{\Omega}, p+1}$ satisfy (114). Then

$$
W_{p+1, k}=\frac{u^{(p+1)}\left(m_{k}\right)}{(p+1) !}+O\left(H^{3 / 2}\right), k=1, \ldots, N .
$$

Proof. From (73), (114)-(115) and (121) it follows that

$$
\begin{aligned}
\hat{A}\left(U^{p+1}-U_{I}^{p+1}, \psi_{p+1, k}\right)_{\Omega_{k}}= & \left(\mu\left(U^{p+1}-u\right)^{\prime}, \psi_{p+1, k}^{\prime}\right)_{\Omega_{k}} \\
& +\left(\kappa\left(U^{p+1}-u\right), \psi_{p+1, k}\right)_{\Omega_{k}}, k=1, \ldots, N .
\end{aligned}
$$

This equation implies that on $\Omega_{k}$,

$$
\begin{aligned}
\rho\left(m_{k}\right) & \times\left|\int_{x_{k-1}}^{x_{k}}\left(U^{p+1}(x)-U_{I}^{p+1}(x)\right)^{\prime \prime} \psi_{p+1, k}^{\prime \prime}(x) d x\right| \\
\leq & \left|\int_{x_{k-1}}^{x_{k}}\left(\rho(x)-\rho\left(m_{k}\right)\right)\left(U^{p+1}-U_{I}^{p+1}\right)^{\prime \prime} \psi_{p+1, k}^{\prime \prime}(x) d x\right| \\
& +\left|\int_{x_{k-1}}^{x_{k}} \mu(x)\left(U(x)-U_{I}(x)\right)^{\prime} \psi_{p+1, k}^{\prime}(x) d x\right| \\
& +\left|\int_{x_{k-1}}^{x_{k}} \kappa(x)\left(U(x)-U_{I}(x)\right) \psi_{p+1, k}(x) d x\right| .
\end{aligned}
$$

Bounds are needed for each of the terms in (142). For the left we have

$$
\begin{aligned}
& \rho\left(m_{k}\right)\left|\int_{x_{k-1}}^{x_{k}}\left(U^{p+1}(x)-U_{I}^{p+1}(x)\right)^{\prime \prime} \psi_{p+1, k}^{\prime \prime}(x) d x\right| \\
& =\rho\left(m_{k}\right) \mid \int_{x_{k-1}}^{x_{k}}\left(U(x)-U_{I}(x)\right)^{\prime \prime} \psi_{p+1, k}^{\prime \prime}(x) d x \\
& \quad+\left(W_{p+1, k}-\bar{W}_{p+1, k}\right) \int_{x_{k-1}}^{x_{k}}\left(\psi_{p+1, k}^{\prime \prime}(x)\right)^{2} d x \mid \\
& =\rho\left(m_{k}\right) \frac{h_{k}^{2 p-1}}{2 p-1}\left(\frac{(p+1) !(p-1) !}{(2 p-2) !}\right)^{2}\left|W_{p+1, k}-\bar{W}_{p+1, k}\right|,
\end{aligned}
$$

where we have used the orthogonality of the Legendre polynomials to show that the first integral on the right is zero.

The Cauchy-Schwarz inequality, (19), (80), (89) and (113) imply that the first integral on the right of (142) yields

$$
\begin{aligned}
& \left|\int_{x_{k-1}}^{x_{k}}\left(\rho(x)-\rho\left(m_{k}\right)\right)\left(U^{p+1}-U_{I}^{p+1}\right)^{\prime \prime} \psi_{p+1, k}^{\prime \prime}(x) d x\right| \\
(144) & \left.\leq\left(\int_{x_{k-1}}^{x_{k}}\left(\rho(x)-\rho\left(m_{k}\right)\right)^{2}\left(\left(U^{p+1}-U_{I}^{p+1}\right)^{\prime \prime}\right)^{2} d x\right)\right)^{1 / 2}\left(\int_{x_{k-1}}^{x_{k}}\left(\psi_{p+1, k}^{\prime \prime}(x)\right)^{2} d x\right)^{1 / 2} \\
& \leq C h_{k}^{p+1 / 2}\left(\left\|U^{\prime \prime}-U_{I}^{\prime \prime}\right\|_{0}+h_{k}^{p+1 / 2}\left|W_{p+1, k}-\bar{W}_{p+1, k}\right|\right) \\
& \leq C H^{2 p+1 / 2}+C h_{k}^{2 p}\left|W_{p+1, k}-\bar{W}_{p+1, k}\right| .
\end{aligned}
$$


The second integral on the right of (142) satisfies

$$
\left|\int_{x_{k-1}}^{x_{k}} \mu(x)\left(U(x)-U_{I}(x)\right)^{\prime} \psi_{p+1, k}^{\prime}(x) d x\right| \leq C H^{p} h_{k}^{p+1 / 2} \leq C H^{2 p+1 / 2},
$$

where we have used the Cauchy-Schwarz inequality, (18) and (81) with $s=1$. Finally again using Cauchy-Schwarz in the third integral gives

$$
\left|\int_{x_{k-1}}^{x_{k}} \kappa(x)\left(U(x)-U_{I}(x)\right) \psi_{p+1, k}(x) d x\right| \leq C H^{p+1} h_{k}^{p+3 / 2} \leq C H^{2 p+5 / 2},
$$

using (17) and (81) with $s=0$. Combining (142)-(146) results in

$$
\rho\left(m_{k}\right) \frac{h_{k}^{2 p-1}}{2 p-1}\left(\frac{(p+1) !(p-1) !}{(2 p-2) !}\right)^{2}\left(1-C h_{k}\right)\left|W_{p+1, k}-\bar{W}_{p+1, k}\right| \leq C H^{2 p+1 / 2}
$$

or

$$
\left|W_{p+1, k}-\bar{W}_{p+1, k}\right| \leq C H^{3 / 2} .
$$

Then (140) follows from (122) and (148).

Theorem 18. Let $u \in H_{0}^{p+5}(\Omega)$ be the solution of (73) and let $U^{p+2} \in S_{0}^{\Delta_{\Omega}, p+2}$ satisfy (117). Then

$$
W_{p+2, k}=\frac{u^{(p+2)}\left(m_{k}\right)}{(p+2) !}+O\left(H^{1 / 2}\right), k=1, \ldots, N .
$$

Proof. From (73), (116)-(117) and (121) we obtain

$$
\begin{aligned}
\hat{A}\left(U^{p+2}-U_{I}^{p+2}, \psi_{p+2, k}\right)_{\Omega_{k}}= & \left(\mu\left(U^{p+1}-u\right)^{\prime}, \psi_{p+2, k}^{\prime}\right)_{\Omega_{k}} \\
& +\left(\kappa\left(U^{p+1}-u\right), \psi_{p+2, k}\right)_{\Omega_{k}}, k=1, \ldots, N
\end{aligned}
$$

Equation (150) implies that

$$
\begin{aligned}
\rho\left(m_{k}\right) \mid & \int_{x_{k-1}}^{x_{k}}\left(U^{p+2}(x)-U_{I}^{p+2}(x)\right)^{\prime \prime} \psi_{p+2, k}^{\prime \prime}(x) d x \mid \\
\leq & \left|\int_{x_{k-1}}^{x_{k}}\left(\rho(x)-\rho\left(m_{k}\right)\right)\left(U^{p+2}-U_{I}^{p+2}\right)^{\prime \prime} \psi_{p+2, k}^{\prime \prime}(x) d x\right| \\
& +\left|\int_{x_{k-1}}^{x_{k}} \mu(x)\left(U(x)-U_{I}(x)\right)^{\prime} \psi_{p+2, k}^{\prime}(x) d x\right| \\
& +\left|\int_{x_{k-1}}^{x_{k}} \kappa(x)\left(U(x)-U_{I}(x)\right) \psi_{p+2, k}(x) d x\right| .
\end{aligned}
$$


As before, bounds are needed for each of the terms in (151). For the term on the left,

$$
\begin{aligned}
& \rho\left(m_{k}\right)\left|\int_{x_{k-1}}^{x_{k}}\left(U^{p+2}(x)-U_{I}^{p+2}(x)\right)^{\prime \prime} \psi_{p+2, k}^{\prime \prime}(x) d x\right| \\
& =\rho\left(m_{k}\right) \mid \int_{x_{k-1}}^{x_{k}}\left(U(x)-U_{I}(x)\right)^{\prime \prime} \psi_{p+2, k}^{\prime \prime}(x) d x \\
& \quad+\left(W_{p+1, k}-\bar{W}_{p+1, k}\right) \int_{x_{k-1}}^{x_{k}} \psi_{p+1, k}^{\prime \prime}(x) \psi_{p+2, k}^{\prime \prime}(x) d x \\
& \quad+\left(W_{p+2, k}-\bar{W}_{p+2, k}\right) \int_{x_{k-1}}^{x_{k}}\left(\psi_{p+2, k}^{\prime \prime}(x)\right)^{2} d x \mid \\
& =\rho\left(m_{k}\right) \frac{h_{k}^{2 p+1}}{2 p+1}\left(\frac{(p+2) ! p !}{(2 p) !}\right)^{2}\left|W_{p+2, k}-\bar{W}_{p+2, k}\right|,
\end{aligned}
$$

where we have used the orthogonality of the Legendre polynomials to show that the first two integrals on the right vanish.

Equations (19), (80), (89), (116) and (148) together with the Cauchy-Schwarz inequality imply that the first integral on the right gives

$$
\begin{aligned}
& \left|\int_{x_{k-1}}^{x_{k}}\left(\rho(x)-\rho\left(m_{k}\right)\right)\left(U^{p+2}-U_{I}^{p+2}\right)^{\prime \prime} \psi_{p+2, k}^{\prime \prime}(x) d x\right| \\
& \left.\quad \leq\left(\int_{x_{k-1}}^{x_{k}}\left(\rho(x)-\rho\left(m_{k}\right)\right)^{2}\left(\left(U^{p+2}-U_{I}^{p+2}\right)^{\prime \prime}\right)^{2} d x\right)\right)^{1 / 2}\left(\int_{x_{k-1}}^{x_{k}}\left(\psi_{p+2, k}^{\prime \prime}(x)\right)^{2} d x\right)^{1 / 2} \\
& \quad \leq C h_{k}^{p+3 / 2}\left(\left\|U^{\prime \prime}-U_{I}^{\prime \prime}\right\|_{0}+h_{k}^{p-1 / 2}\left|W_{p+1, k}-\bar{W}_{p+1, k}\right|+h_{k}^{p+1 / 2}\left|W_{p+2, k}-\bar{W}_{p+2, k}\right|\right) \\
& \quad \leq C H^{2 p+3 / 2}+C h_{k}^{2 p+1}\left|W_{p+1, k}-\bar{W}_{p+1, k}\right|+C h_{k}^{2 p+2}\left|W_{p+2, k}-\bar{W}_{p+2, k}\right| \\
& \quad \leq C H^{2 p+3 / 2}+C h_{k}^{2 p+2}\left|W_{p+2, k}-\bar{W}_{p+2, k}\right| .
\end{aligned}
$$

The second integral on the right of (151) yields

$$
\left|\int_{x_{k-1}}^{x_{k}} \mu(x)\left(U(x)-U_{I}(x)\right)^{\prime} \psi_{p+2, k}^{\prime}(x) d x\right| \leq C H^{p} h_{k}^{p+3 / 2} \leq C H^{2 p+3 / 2},
$$

where we have used the Cauchy-Schwarz inequality, (18) and (81) with $s=1$. Finally, again using Cauchy-Schwarz in the third integral, gives

$$
\left|\int_{x_{k-1}}^{x_{k}} \kappa(x)\left(U(x)-U_{I}(x)\right) \psi_{p+2, k}(x) d x\right| \leq C H^{p+1} h_{k}^{p+5 / 2} \leq C H^{2 p+7 / 2},
$$

using (17) and (81) with $s=0$. Combining (151)-(155) results in

$$
\rho\left(m_{k}\right) \frac{h_{k}^{2 p+1}}{2 p+1}\left(\frac{(p+2) ! p !}{(2 p) !}\right)^{2}\left(1-C h_{k}\right)\left|W_{p+2, k}-\bar{W}_{p+2, k}\right| \leq C H^{2 p+3 / 2}
$$

or

$$
\left|W_{p+2, k}-\bar{W}_{p+2, k}\right| \leq C H^{1 / 2} .
$$

Then (149) follows from (130) and (157). 
Corollary 19. Under the hypotheses of Lemma 7 and letting $U^{p+1}$ and $U^{p+2}$ satisfy (114) and (117), respectively, for $k=1, \ldots, N$,

$$
\frac{\left\|U-U^{p+1}\right\|_{2}}{\|u-U\|_{2}}-1 \leq O(H), \frac{\left\|U^{p+1}-U^{p+2}\right\|_{2}}{\left\|u-U^{+}\right\|_{2}}-1 \leq O\left(H^{1 / 2}\right) .
$$

Proof. The proof follows from Corollary 5, Lemma 9 and Theorems 17 and 18.

The same result holds in the time-dependent case.

Corollary 20. Under the hypotheses of Lemma 10 and letting $U^{p+1}$ and $U^{p+2}$ satisfy (118) and (119), respectively, for $k=1, \ldots, N$, then

$$
\frac{\left\|U-U^{p+1}\right\|_{2}}{\|u-U\|_{2}}-1 \leq O(H), \frac{\left\|U^{p+1}-U^{p+2}\right\|_{2}}{\left\|u-U^{+}\right\|_{2}}-1 \leq O\left(H^{1 / 2}\right) .
$$

Proof. With slight modifications the proofs of Theorems 17 and 18 can be extended to the time-dependent case using Lemma 10 and Corollary 13 since the only additional term in the inequalities (142) and (151) is

$$
\left|\int_{x_{k-1}}^{x_{k}}\left(u_{t}-U_{t}\right) \psi_{l, k}(x) d x\right|,
$$

where $l=p+1$ and $p+2$, respectively. Appropriate bounds on (160) are obtained via Cauchy-Schwarz, (17) and (87). The proof then follows along the same lines as the proof of Corollary 19.

We now explain briefly how the results of this section can be used to guide adaptivity. On each element $\Omega_{k}$ we compute the error indicators $E_{0, k} \equiv \mid U-$ $\left.U^{p_{k}+1}\right|_{2, k}$ and $E_{1, k} \equiv\left|U^{p_{k}+1}-U^{p_{k}+2}\right|_{2, k}$ together with $E_{s, k} \equiv\left|U_{p_{k}-s, k} \phi_{p_{k}-s, k}\right|_{2, k}$, $s=-1,-2$. The latter indicators estimate errors in the next two lower-order finite element solutions. These indicators can be used to control $h$-, $p$ - or $h p$-refinement strategies. Adaptive codes must first decide whether or not to modify the grid and in the case of modification what changes to make. For steady-state problems the grid is modified if

$$
E_{r m s} \equiv \frac{\left(\sum_{k=1}^{N} E_{0, k}^{2}\right)^{1 / 2}}{\text { atol }+ \text { rtol }\|U\|_{2}}>1,
$$

where atol and rtol are user-prescribed absolute and relative error tolerances, respectively. For time-dependent problems this criterion is insufficient as it will not allow coarsening when the error is below the tolerance. In this case an effective rule depends on the number of elements to be refined $\left(N_{r}\right)$ or coarsened $\left(N_{c}\right)$. An element $k$ is marked for refinement or coarsening, respectively, if 26

$$
\frac{E_{s, k}}{\text { atol }+ \text { rtol }\|U\|_{2}}>0.8 / \sqrt{N}, \frac{E_{s, k}}{\text { atol }+ \text { rtol }\|U\|_{2}}<0.2 /\left(2^{p_{k}} \sqrt{N}\right) .
$$

The grid is modified if $N_{r}>0$ or $E_{r m s}<0.1$ or $N_{c} / N>0.6$ [26]. The first is a refinement criterion and the second two promote coarsening.

Decisions on how to adapt the grid also depend on the marking scheme. For $h$-adaptivity we use (162) with $s=0$; the other indicators are not needed. With $p$-adaptivity, (162) with $s=0$ is used for refinement (increasing the order by one). Coarsening (decreasing the order by one) also makes use of the indicator with $s=-1$. In addition we constrain the grids by imposing (63) and (64) when using $h$ and $p$-refinement, respectively, as described in section 3 . The $h p$-refinement strategy 
consists of four stages. Stage 1 involves choosing a new order $\hat{p}_{k} \in\left\{p_{k}-1, p_{k}, p_{k}+1\right\}$ on each element depending on trends in the error indicators $E_{s, k}$ with $s=-2, \ldots, 1$ 21. In the second stage new elements are created if the error estimate on an element still violates the first equation in (162), where the indicator $E_{0, k}$ is replaced by $E_{-1, k}$ or $E_{1, k}$ if $\hat{p}_{k}$ is one less or one more than $p_{k}$, respectively. Simultaneously (63) is enforced. In Stage 3 elements sharing the same parent are coalesced if the errors on both satisfy the second equation of (162) and if (63) is not violated. Finally (64) and other order smoothing operations [26] are imposed. More details can be found in $[20,21,26]$.

\section{Computational Results}

The codes, written in Fortran 90, can be run in either double or quadruple precision. For both steady-state and time-dependent problems the codes begin with a uniform base grid containing $N_{b}$ elements of order $p_{b}$. The default values of $N_{b}$ and $p_{b}$ are 20 and 5, respectively. For uniform grids, $N=N_{b}$ and $p=p_{b}$.

Example 5.1. Consider the linear steady-state equation

$$
f(x)=-u+u_{x x}-u_{x x x x}, 0 \leq x \leq 1,
$$

where Dirichlet boundary conditions and $f$ are chosen so that the exact solution is

$$
u(x)=\tanh (20(x-0.55)) .
$$

We begin by providing computational evidence of Corollary 19. We solved (163) on a sequence of uniform grids with $p=3, \ldots, 6$ and $h=1 / N, N=5,10,20,40$ and 80. Estimator accuracy was measured using the effectivity index, the ratio of the estimated to the true error $e=u-U$. In particular we computed the effectivity indexes

$$
\theta=\frac{\left\|U-U^{p+1}\right\|_{2}}{\|u-U\|_{2}}, \theta^{+}=\frac{\left\|U^{p+1}-U^{p+2}\right\|_{2}}{\left\|u-U^{+}\right\|_{2}} .
$$

TABLE 1. Finite element errors and effectivity indexes $\theta$ and $\theta^{+}$ of order $p$ and $p+1$, respectively, obtained in solving (163) on uniform grids with $N$ elements and order $p$.

\begin{tabular}{|c|c|c|c|c|c|c|c|c|}
\hline$N$ & $p$ & $\|e\|_{2}$ & $\theta$ & $\theta^{+}$ & $p$ & $\|e\|_{2}$ & $\theta$ & $\theta^{+}$ \\
\hline 5 & 3 & $11.8 \times 10^{2}$ & 0.767 & 0.156 & 4 & $10.0 \times 10^{2}$ & 0.156 & 0.549 \\
\hline 10 & 3 & $2.57 \times 10^{1}$ & 0.416 & 0.983 & 4 & $2.33 \times 10^{1}$ & 0.983 & 0.985 \\
\hline 20 & 3 & $1.60 \times 10^{1}$ & 0.998 & 0.641 & 4 & 1.09 & 0.641 & 0.987 \\
\hline 40 & 3 & 3.36 & 0.993 & 0.991 & 4 & $4.06 \times 10^{-1}$ & 0.991 & 0.998 \\
\hline 80 & 3 & $8.54 \times 10^{-1}$ & 0.998 & 0.998 & 4 & $5.42 \times 10^{-2}$ & 0.998 & 0.999 \\
\hline 5 & 5 & $5.21 \times 10^{1}$ & 0.549 & 0.492 & 6 & $4.47 \times 10^{1}$ & 0.492 & 0.0454 \\
\hline 10 & 5 & 4.29 & 0.0985 & 0.989 & 6 & 4.27 & 0.989 & 0.145 \\
\hline 20 & 5 & $8.35 \times 10^{-1}$ & 0.987 & 0.989 & 6 & $1.34 \times 10^{-1}$ & 0.989 & 0.935 \\
\hline 40 & 5 & $5.33 \times 10^{-2}$ & 0.998 & 0.983 & 6 & $3.77 \times 10^{-3}$ & 0.983 & 1.000 \\
\hline 80 & 5 & $3.12 \times 10^{-3}$ & 0.999 & 0.999 & 6 & $1.68 \times 10^{-4}$ & 0.999 & 0.999 \\
\hline
\end{tabular}


The results in Table 1 show that the estimators converged to the true error as the grids were refined as predicted by the theory even before the errors were in the asymptotic range.

TABLE 2. Estimates of the error in $H^{2}$ and the convergence rates of $\theta$ and $\theta^{+}$to 1 , respectively, in solving (163) on four uniform grids with $p=3,4$ and $h=1 / N, N=40,80,160$ and 320 .

\begin{tabular}{|c|c|c|c|c|}
\hline$N$ & $p$ & $\|e\|_{2}$ & $|\theta-1|$ & $\left|\theta^{+}-1\right|$ \\
\hline 40 & 3 & 3.36 & $7.30 \times 10^{-3}$ & $8.66 \times 10^{-3}$ \\
\hline 80 & 3 & $8.54 \times 10^{-1}$ & $2.02 \times 10^{-3}$ & $1.65 \times 10^{-3}$ \\
\hline 160 & 3 & $2.14 \times 10^{-1}$ & $5.06 \times 10^{-4}$ & $4.14 \times 10^{-4}$ \\
\hline 320 & 3 & $2.14 \times 10^{-1}$ & $1.27 \times 10^{-4}$ & $1.04 \times 10^{-4}$ \\
\hline 40 & 4 & $4.06 \times 10^{-1}$ & $8.66 \times 10^{-3}$ & $2.50 \times 10^{-3}$ \\
\hline 80 & 4 & $5.43 \times 10^{-2}$ & $1.65 \times 10^{-3}$ & $1.45 \times 10^{-3}$ \\
\hline 160 & 4 & $6.84 \times 10^{-3}$ & $4.14 \times 10^{-4}$ & $3.63 \times 10^{-4}$ \\
\hline 320 & 4 & $8.56 \times 10^{-4}$ & $1.04 \times 10^{-4}$ & $9.09 \times 10^{-5}$ \\
\hline
\end{tabular}

A more detailed analysis for $p=3$ and 4 is provided in Table 2. Here we computed $\theta-1$ and $\theta^{+}-1$ on four grids with $h=1 / N$ and $N=40,80,160$ and 320 . For both $\theta$ and $\theta^{+}$the convergence is $O\left(H^{2}\right)$, which is better than the theory predicts. Comparable results were obtained when $\rho(x)=1+x$ while modifying $f(x)$ to maintain (164) as a solution. That the convergence rate is $H^{2}$ is suggested by a closer examination of the interpolant error (48). In particular using the properties of Legendre polynomials it is possible to show that the second term on the right side of (60) is $O\left(h^{2 p+2}\right)$.

We also solved (163) using the three adaptive strategies, $h$ - $p$ - and $h p$-adaptivity with uniform grids as a benchmark for three tolerances atol $=10^{-m}, m=3,5$ and $7, r t o l=0$. The total number of unknowns $N_{d o f}$, CPU time and levels of refinement $N_{l v l}$, together with the exact error, effectivity index and number of unknowns on the final grid $N_{\text {dof }}^{f}$ are displayed in Table 3.

In all cases the tolerance is met. The uniform grid and $p$-refinement strategies required user intervention to achieve this. In the case of uniform grids the results were obtained by computing on a sequence of grids with increasing $N_{b}$ until the tolerance was satisfied. The additional time needed for these trials is not reflected in the CPU times in Table 3. It is therefore more appropriate to compare $N_{d o f}$ for the uniform grids with $N_{d o f}^{f}$ for the adaptive grids. By this measure the adaptive strategies lead to the use of fewer degrees of freedom than when employing uniform grids. The times for both $p$ - and $h p$-adaptivity are also smaller than for the uniform grid computations. We conclude that adaptivity pays off, even accounting for the fact that the errors on the final grids are not the same.

$P$-adaptivity is also superior to $h$-adaptivity, although the code is limited by a maximum order, 14. As a result for the smallest tolerance, atol $=10^{-7}$, user intervention was required. Specifically, it was necessary to increase $N_{b}$ so that the tolerance could be met. We chose $N_{b}=30$ for this case. This scenario was also used to demonstrate that (64) is essential to the performance of $p$-adaptivity. When the $p$-adaptive code was run without imposing (64) it generated a solution for which 
TABle 3. Total number of unknowns $N_{d o f}$, CPU time and levels of refinement $N_{l v l}$ together with the errors $\|e\|_{2}$, effectivity indexes and number of unknowns on the final grid, $\left(N_{d o f}^{f}\right)$, in solving (163) for three tolerances atol $=10^{-m}, m=3,5,7$ using, from top to bottom, uniform, $h-, p$ - and $h p$-adaptive grids.

\begin{tabular}{|c|c|c|c|c|c|}
\hline \multicolumn{7}{|c|}{ uniform grid } \\
\hline atol & $N=N_{\text {dof }}=N_{\text {dof }}^{f}$ & time (in sec) & $N_{\text {lvl }}$ & $\|e\|_{2}$ & $\theta$ \\
\hline $10^{-3}$ & 442 & 0.0330 & 1 & $8.78 \times 10^{-4}$ & 0.999 \\
\hline $10^{-5}$ & 1802 & 0.0900 & 1 & $3.15 \times 10^{-6}$ & 1.000 \\
\hline $10^{-7}$ & 5202 & 0.3829 & 1 & $4.53 \times 10^{-8}$ & 1.000 \\
\hline \multicolumn{5}{|c|}{$h$-adaptive grid } \\
\hline atol & $N_{\text {dof }}\left(N_{\text {dof }}^{f}\right)$ & time (in sec) & $N_{\text {lvl }}$ & $\|e\|_{2}$ & $\theta$ \\
\hline $10^{-3}$ & $600(226)$ & 0.0330 & 4 & $2.69 \times 10^{-4}$ & 0.999 \\
\hline $10^{-5}$ & $1956(658)$ & 0.1040 & 6 & $3.13 \times 10^{-6}$ & 0.999 \\
\hline $10^{-7}$ & $4922(1774)$ & 0.2810 & 7 & $5.30 \times 10^{-8}$ & 1.000 \\
\hline \multicolumn{7}{|c|}{$p$-adaptive grid } \\
\hline atol & $N_{\text {dof }}\left(N_{\text {dof }}^{f}\right)$ & time (in sec) & $N_{l v l}$ & $\|e\|_{2}$ & $\theta$ \\
\hline $10^{-3}$ & $490(117)$ & 0.0290 & 5 & $3.12 \times 10^{-4}$ & 0.640 \\
\hline $10^{-5}$ & $1033(177)$ & 0.0590 & 8 & $4.66 \times 10^{-6}$ & 0.961 \\
\hline $10^{-7}$ & $1747(257)$ & 0.1020 & 9 & $5.13 \times 10^{-8}$ & 0.929 \\
\hline \multicolumn{7}{|c|}{$h p$-adaptive grid } \\
\hline atol & $N_{\text {dof }}\left(N_{d o f}^{f}\right)$ & time (in sec) & $N_{l v l}$ & $\|e\|_{2}$ & $\theta$ \\
\hline $10^{-3}$ & $545(179)$ & 0.0290 & 4 & $1.77 \times 10^{-4}$ & 0.998 \\
\hline $10^{-5}$ & $1040(316)$ & 0.0550 & 5 & $2.83 \times 10^{-6}$ & 0.998 \\
\hline $10^{-7}$ & $1499(531)$ & 0.0820 & 5 & $8.28 \times 10^{-8}$ & 1.000 \\
\hline \multicolumn{7}{|c|}{}
\end{tabular}

$E_{r m s}<1$, i.e., for which the error estimate was smaller than the tolerance, $10^{-7}$, but for which the actual error in $H^{2}$ was $2.3 \times 10^{-2}$.

The performance of the $h p$-adaptive code is comparable to the $p$-adaptive version. $H p$-refinement required more degrees of freedom overall and significantly more on the final grid. However, it used less time than the $p$-adaptive code since it required fewer refinement levels to meet the tolerance (each refinement level requires matrix assembly and factorization). It was also more robust than $p$-adaptivity (as was $h$-adaptivity) with effectivity indexes much closer to 1 . Additionally no user intervention was required.

As noted in the introduction we use a method-of-lines approach to solve timedependent problems. This familiar strategy [37] first discretizes (3) in space and solves the resulting system of ordinary differential equations with an appropriate time integrator. We use the multistep code dassl [7]. The time integrator interfaces with the spatial discretization in two ways. Separate temporal error tolerances abs_tol and rel_tol must be provided to dassl. In MOL codes it is typical that [19, 36] abs_tol $=\tau \times$ atol and rel_tol $=\tau \times$ rtol. Second, the error indicators $E_{s, k}$ and estimator $E_{r m s}$ are computed and the grid potentially modified after every $M$ successful steps of dassl. We took $\tau=0.002$ and $M=5$ [26]. The initial grid was 

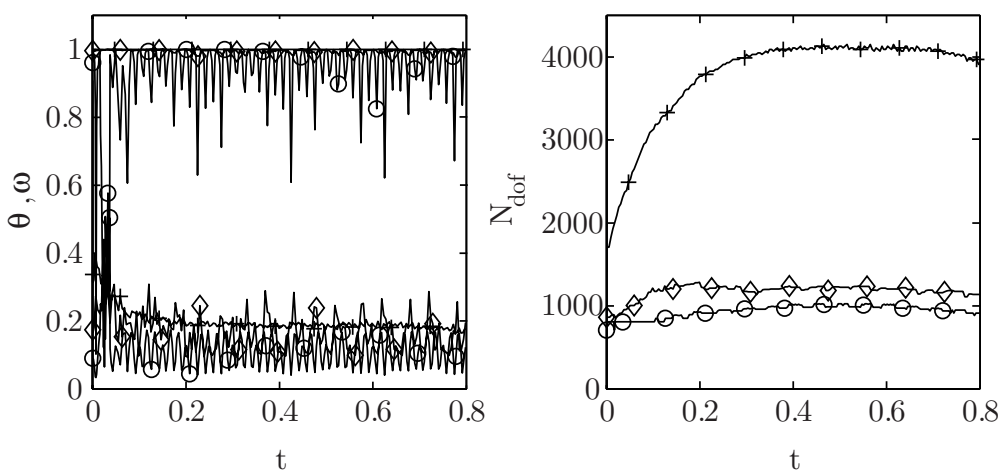

Figure 1. The effectivity index $\theta(t)$ and error index $\omega(t)$ (left) and number of degrees of freedom $N_{\text {dof }}(t)$ (right) in solving (166) using $h_{-}(+), p-(\circ)$, and $h p-(\diamond)$ adaptivity with atol $=10^{-5}$ and $r$ tol $=0$.

generated by refining the base grid until the interpolation error in $H^{2}$ of the initial conditions satisfies the user tolerances.

Example 5.2. Consider the linear time-dependent equation

$$
u_{t}+f(x, t)=-u+u_{x x}-u_{x x x x}, 0 \leq x \leq 1, t>0,
$$

where initial, Dirichlet boundary conditions and $f$ are chosen so that the exact solution is

$$
u(x, t)=\tanh (20(x-t)) .
$$

We solved (166) for $t \in(0,0.8]$ using the three adaptive strategies and atol $=10^{-5}$, $r t o l=0$. To examine reliability we computed the effectivity index $\theta(t)$ and error index $\omega(t)=\frac{\|e\|_{2}}{\text { atol }}$. Ideally both should be close to one with $\omega(t)<1$. The values of these indexes are shown on the left in Figure 1. As with the steady-state problem the estimator is quite accurate in conjunction with the $h$ - and $h p$-adaptive strategies but is notably poorer with $p$-adaptivity. For all three methods, the error index remains below one with the smoothest performance exhibited by the $h$-refinement code.

Efficiency was measured by the number of degrees of freedom $N_{d o f}(t)$ and the total CPU time. As shown on the right of Figure 1 the $p$-refinement code employs the fewest unknowns with $h p$-refinement a close second while $h$-refinement uses considerably more. The CPU times for the three codes were $8.93 \mathrm{~s}(h), 4.00 \mathrm{~s}(p)$ and 3.41s $(h p)$. Here the $h p$-adaptive version comes out ahead. One reason the $p$-refinement run took longer than the $h p$-refinement is it required a larger number of time steps. This is similar to the effect of the number of levels of refinement in Example 5.1 since it involved additional matrix assembly and factorization.

\section{Conclusions}

A posteriori error estimators for second-order equations have been successfully extended to fourth-order problems. This has been done by creating a hierarchical 
basis from Hermite-Lobatto polynomials. On grids of uniform order, the two estimators required by the adaptive strategy are proved to converge to the true errors at two orders, respectively. Computations show the reliability of the estimators and their ability to guide $h-, p$ - and $h p$-adaptive strategies. For problems with smooth solutions, $p$ - and $h p$-adaptivity perform well with $h p$-refinement having the edge in reliability.

The a posteriori error estimates can be extended in several ways. The proofs in section 4 can be modified to handle certain classes of nonlinearities following [25]. Second we can generate a family of Lobatto polynomials that can lead to asymptotically equivalent interpolants for higher-order equations in one space dimension. Additionally, following the approach taken by Moore [23, the estimators can be extended to two and three space dimensions (as opposed to the method of Adjerid [1, which, for odd order, uses jumps in solution derivatives). Finally superconvergence properties for second-order problems [40] can be extended to fourth-order and higher starting from the results herein.

\section{REFERENCES}

[1] S. Adjerid, A posteriori error estimates for fourth-order elliptic problems, Comput. Meth. Appl. Mech. Engrg. 191, (2002), 2539-2559. MR1902705 (2003j:65105)

[2] S. Adjerid, P.K. Moore and J. Teresco, eds., ADAPT'03: Adaptive methods for partial differential equations and large-scale computation, Appl. Numer. Math. 52, (2005). MR2116907

[3] S. Adjerid, I. Babǔska and J.E. Flaherty, A posteriori error estimation for the finite element method-of-lines solution of parabolic problems, Math. Model. Meth. Appl. Sci. 9, (1999), 261-286. MR1674560(2000a:65117)

[4] M. Ainsworth and J.T. Oden, A Posteriori Error Estimation in Finite Element Analysis, Wiley/Interscience, New York, 2000. MR.1885308 (2003b:65001)

[5] I. Babǔska, J.E. Flaherty, W.D. Henshaw, J.E. Hopcroft, J.E. Oliger and T. Tezduyar, eds., Modeling, Mesh Generation, and Adaptive Numerical Methods for Partial Differential Equations, Springer-Verlag, New York, 1995. MR.1370242 (96g:65002)

[6] J.B. van den Berg and R.C. Vandervorst, Stable patterns for fourth-order parabolic equations, Duke Math. J. 115, (2002), 513-558. MR.1940411 (2003j:35136)

[7] K.E. Brenan, S.L. Campbell and L.R. Petzold, Numerical Solution of Initial Value Problems in Differential-Algebraic Equations, North-Holland, New York, $1989 . \quad$ MR.1101809 (92e:65001)

[8] C.J. Budd and R. Kuske, Localized periodic patterns for the nonsymmetric generalized SwiftHohenberg equation, Phys. D 208, (2005), 73-95. MR2167908 (2007d:35141)

[9] J. Cahn and J. Hilliard, Free energy of a nonuniform system. I. Interfacial free energy, $J$. Chem. Phys. 28, (1958), 258-267.

[10] Y. Cheng and C.-W. Shu, A discontinuous Galerkin finite element method for time dependent partial differential equations with higher order derivatives, Math. Comput. 77, (2008), 699730. MR2373176 (2008m:65252)

[11] P. Cvitanovic, R.L. Davidchack and E. Siminos, State space geometry of a spatio-temporally chaotic Kuramoto-Sivashinsky flow, arXiv:0709.2944v1, (2007), 1-27.

[12] G.T. Dee and W. van Saarloos, Bistable systems with propagating fronts leading to pattern formation, Phys. Rev. Lett. 60, (1988), 2641-2644.

[13] L. Demkowicz, Computing with hp-Adaptive Finite Elements. Volume 1. One and Two Dimensional Elliptic and Maxwell Problems, Chapman \& Hall/CRC, Boca Raton, 2007. MR2267112 (2007k:65003)

[14] D.J. Eyre, Systems of Cahn-Hilliard equations, SIAM J. Appl. Math. 53, (1993), 1686-1712. MR.1247174 (94j:73010)

[15] A. Ern and J.-L. Guermond, Theory and Practice of Finite Elements, Springer, New York, 2004. MR2050138(2005d:65002)

[16] M. Gameiro, K. Mischaiskow and T. Wanner, Evolution of pattern complexity in the CahnHilliard theory of phase separation, Acta Materialia 53, (2005), 693-704. 
[17] M'F. Hilali, S. Métens, P. Borckmans and G. Dewel, Pattern selection in the generalized Swift-Hohenberg model, Phys. Rev. E 31, (1995), 2046-2055.

[18] Y. Kuramoto and T. Tsuzuki, Persistent propagation of concentration waves in dissipative media far from thermal equilibrium, Progress Theor. Phys. 55, (1976), 356-368.

[19] J. Lawson, M. Berzins, and P.M. Dew, Balancing space and time errors in the method of lines for parabolic equations, SIAM J. Sci. Stat. Comput. 12, (1991), 573-594. MR 1093207 (92a:65252)

[20] P.K. Moore, Comparison of adaptive methods for one-dimensional parabolic systems, Appl. Numer. Math. 16, (1995), 471-488. MR1325260 (95m:65170)

[21] P.K. Moore, Interpolation error-based a posteriori error estimation for two-point boundary value problems and parabolic equations in one space dimension, Numer. Math. 90, (2001), 149-177. MR.1868766 (2002h:65111)

[22] P.K. Moore, Applications of Lobatto polynomials to an adaptive finite element method: a posteriori error estimates for $h p$-adaptivity and grid-to-grid interpolation, Numer. Math. 94, (2003), 367-401. MR1974560 (2004e:65105)

[23] P.K. Moore, Implicit interpolation error-based error estimation for reaction-diffusion equations in two space dimensions, Comput. Meth. Appl. Mech. Engrg. 192, (2003), 4379-4401. MR2008075 (2004i:65107)

[24] P.K. Moore, Interpolation error-based a posteriori error estimation for $h p$-refinement using first and second derivative jumps, Appl. Numer. Math. 48, (2004), 63-82. MR2027822 (2004k:65122)

[25] P.K. Moore, An implicit interpolation error-based error estimation strategy for $h p$-adaptivity in one space dimension, J. Numer. Math. 12, (2004), 143-167. MR2062583 (2005b:65087)

[26] P.K. Moore, HP4: a $h p$-adaptive finite element code for solving fourth-order equations in one space dimension, preprint.

[27] J.T. Oden and G.F. Carey, Finite Elements. Mathematical Aspects. Volume IV, Prentice Hall, Inc., Englewood Cliffs, NJ, 1983. MR767806 (86m:65001d)

[28] E.D. Rainville, Special Functions, Chelsea Publishing Co., New York, 1960. MR0107725 (21:6447)

[29] M. Rangelova, Error estimation for fourth order partial differential equations, Ph.D. Thesis, Southern Methodist University, Dallas, TX, 2007.

[30] G.I. Sivashinsky, Nonlinear analysis of hydrodynamic instability in laminar flames, I. Acta Astronautica 4, (1977), 1177-1206. MR0502829(58:19741)

[31] G. Strang and G.J. Fix, An Analysis of the Finite Element Method, Prentice Hall, Inc., Englewood Cliffs, NJ, 1973. MR0443377 (56:1747)

[32] Y.S. Smyrlis and D.T. Papageorgiou, Computational study of chaotic and ordered solutions of the Kuramoto-Sivashinsky equation, ICASE Report 96-12, (1996), 1-32.

[33] E. Süli and I. Mozolevski, $h p$-version interior penalty DGFEMs for the biharmonic equation, Comput. Meth. Appl. Mech. Engrg. 196, (2007), 1851-1863. MR2298696 (2008c:65350)

[34] R. Verfürth, A Review of a Posteriori Error Estimation and Adaptive Mesh-Refinement Techniques, Wiley-Teubner, Chichester, 1996.

[35] R. Wait and A.R. Mitchell, Finite Element Analysis and Applications, John Wiley \& Sons, Chichester, 1985. MR817440(87i:65192)

[36] R. Wang, P. Keast, and P. Muir, A high-order global spatially adaptive collocation method for 1-D parabolic PDEs, Appl. Numer. Math. 50, (2004), 239.250. MR2066739

[37] A. Vande Wouwer, Ph. Saucez and W.E. Schiesser, eds., Adaptive Method of Lines, Chapman \& Hall/CRC, Boca Raton, 2001. MR 1851429 (2002c:65004)

[38] D. Yu, Asymptotically exact a-posteriori error estimator for elements of bi-even degree, Math. Numer. Sinica 19, (1991), 89-101.

[39] D. Yu, Asymptotically exact a-posteriori error estimator for elements of bi-odd degree, Math. Numer. Sinica 19, (1991), 307-314.

[40] Z. Zhang, Superconvergence of spectral collocation and $p$-version methods in one dimensional problems, Math. Comput. 74, (2005), 1621-1636. MR.2164089 (2006h:65199)

Department of Mathematics, Southern Methodist University, Dallas, Texas 75275

eVerge Group, Plano, Texas 75093 